\title{
Nonlinear realization of chiral symmetry breaking in holographic soft wall models
}

\author{
Alfonso Ballon-Bayona ${ }^{1, *}$ and Luis A. H. Mamani ${ }^{2, \dagger}$ \\ ${ }^{1}$ Instituto de Física, Universidade Federal do Rio de Janeiro, \\ Caixa Postal 68528, Rio de Janeiro 21941-972, Brazil \\ ${ }^{2}$ Centro de Ciências Naturais e Humanas, Universidade Federal do ABC, \\ Rua Santa Adélia 166, 09210-170 Santo André, São Paulo, Brazil
}

(Received 16 February 2020; accepted 22 June 2020; published 13 July 2020)

\begin{abstract}
We investigate nonlinear extensions of the holographic soft wall model proposed by Karch et al. [Phys. Rev. D 74, 015005 (2006)] with a positive quadratic dilaton. We consider a Higgs potential for the tachyonic field that brings a more natural realization of chiral symmetry breaking in the infrared regime. Utilizing the AdS/CFT dictionary and holographic renormalization, we find the chiral condensate as a function of the quark mass. The nonlinearity of the Higgs potential leads to a nonlinear relation between the chiral condensate and the quark mass. Solving the effective Schrödinger equations for the field perturbations, we estimate meson masses and decay constants and evaluate their dependence on the quark mass. In the axial and pseudoscalar sector we find an interesting behavior for the decay constants as the quark mass increases. We also investigate the effect of a five-dimensional running mass for the tachyonic field. We conclude that nonlinear soft wall models with a Higgs potential for the tachyon and a positive quadratic dilaton do not provide spontaneous symmetry breaking in the chiral limit.
\end{abstract}

DOI: $10.1103 /$ PhysRevD.102.026013

\section{INTRODUCTION}

The dynamical breaking of chiral symmetry in quantum chromodynamics (QCD) is a fascinating phenomenon that occurs in the strongly coupled (nonperturbative) regime. Chiral symmetry breaking is the mechanism responsible for dynamical generation of quark masses at low energies, and it is crucial for the description of mesons and baryons. The well established order parameter associated with chiral symmetry breaking is the so-called chiral condensate $\langle\bar{q} q\rangle$, which is the vacuum expectation value (VEV) of the quark mass operator. A nonzero chiral condensate signalizes chiral symmetry breaking, and it can be generated by a nontrivial QCD vacuum. The phenomenon of chiral symmetry breaking is closely related to the phenomenon of quark confinement [1].

The traditional approaches used to investigate the nonperturbative regime of QCD are lattice QCD and the Schwinger-Dyson equations. They have provided plenty of insights on the mechanism for dynamical chiral

\footnotetext{
*aballonb@if.ufrj.br

†luis.mamani@ufabc.edu.br
}

Published by the American Physical Society under the terms of the Creative Commons Attribution 4.0 International license. Further distribution of this work must maintain attribution to the author(s) and the published article's title, journal citation, and DOI. Funded by SCOAP ${ }^{3}$. symmetry breaking as well as quantitative predictions for hadronic phenomenology. More recently, a complementary approach to nonperturbative QCD has been developed. The so-called holographic QCD approach builds five-dimensional (5D) gravitational models dual to four-dimensional (4D) nonperturbative quantum field theories similar to QCD. This approach is based on the AdS/CFT correspondence and the gauge/gravity duality.

One of the main achievements of the holographic QCD approach is the discovery of a universal criterion for confinement [2] based on the holographic map between 5D classical strings and 4D Wilson loops [3]. The problem of chiral symmetry breaking is also fascinating in holographic QCD. Since the chiral symmetry group $U\left(N_{f}\right)_{L} \times$ $U\left(N_{f}\right)_{R}$ is a $4 \mathrm{D}$ global symmetry group, it maps to $5 \mathrm{D}$ (local) gauge symmetry group. This implies that 4D chiral symmetry breaking corresponds to 5D (non-Abelian) gauge symmetry breaking. A minimal 5D holographic QCD model for chiral symmetry breaking was proposed in $[4,5]$ and based on the so-called hard wall model [6]. In that approach the breaking of the 5D non-Abelian gauge symmetry is induced by a 5D scalar field $X$ that is dual to the quark mass operator $\bar{q} q$. The AdS/CFT dictionary maps the $5 \mathrm{D}$ squared mass $m_{X}^{2}$ to the conformal dimension of $\bar{q} q$ implying a tachyonic mass $m_{X}^{2}=-3$.

Although the model in $[4,5]$ successfully introduces the quark mass and chiral condensate, it does not provide a dynamical mechanism for chiral symmetry in the sense that 
the chiral condensate is fixed by boundary conditions in the infrared (IR) regime. An alternative approach was proposed in [7] and is known as the soft wall model. That approach was physically motivated by experimental data on the meson spectrum indicating a set of linear Regge trajectories. In holographic QCD meson masses arise as eigenvalues of effective Schrödinger potentials for 5D field perturbations. The soft wall model introduces a smooth cutoff in the form of a background scalar field $\Phi(z)$, known as the dilaton. As shown in [7], a positive quadratic dilaton $\Phi(z) \sim z^{2}$ in the IR leads to approximate linear Regge trajectories for the mesons. In the soft wall model a nontrivial dynamics for the tachyonic field $X$ is driven by the presence of the dilaton field $\Phi(z)$ and, instead of imposing a boundary condition at an arbitrary energy scale, the chiral condensate is obtained from a regularity condition.

Despite providing a more realistic meson spectrum, the original soft wall model leads to a linear realization of chiral symmetry breaking driven by an IR regularity condition. Indeed, the chiral condensate $\langle\bar{q} q\rangle$ is proportional to the quark mass $m_{q}$ just as a consequence of the linearity of the tachyonic field equation. This implies, in particular, that taking the chiral limit $m_{q} \rightarrow 0$ in the soft wall model does not lead to spontaneous chiral symmetry breaking (the chiral condensate vanishes). This differs significantly from QCD, where spontaneous symmetry breaking always occurs in the chiral limit.

In this work we investigate nonlinear extensions of the holographic soft wall model. We consider a 5D Higgs potential for the tachyonic field $X$ leading to a nonlinear differential equation for the tachyonic field. The nonlinearity allows us to find a family of solutions in the IR depending on only one parameter, which we call $C_{0}$. For fixed $C_{0}$ we will be able to solve numerically the tachyon differential equation. Extracting the source and VEV coefficients for the tachyon solution near the boundary, we utilize the AdS/CFT correspondence and map those parameters to the quark mass and chiral condensate, respectively. We will show that the nonlinearity of the tachyon differential equation implies a nonlinear relation between the chiral condensate $\langle\bar{q} q\rangle$ and the quark mass $m_{q}$. We will show, in particular, that for the original Higgs potential the chiral condensate always grows with the quark mass, as expected in QCD; see, i.e., [8]. We will find, however, that near the chiral limit $m_{q} \rightarrow 0$ the nonlinear soft wall models based on a Higgs potential behave in the same way as the original soft wall model, and therefore the chiral condensate vanishes. A similar result was recently found in [9].

In this work we perform a systematic analysis of the background and field perturbations for nonlinear soft wall models based on a positive quadratic dilaton $\Phi$ and a Higgs potential for the tachyonic field $X$. For completeness we consider the cases of negative and positive couplings for the Higgs potential $U(|X|)=m_{X}^{2}|X|^{2}+\lambda|X|^{4}$. A negative coupling $\lambda<0$ never provides a minimum, whereas the positive case $\lambda>0$ corresponds to the original Mexican hat potential leading to a minimum. Our results for the meson spectrum and decay constants support our main conclusions and also bring some pleasant surprises. In particular, we find an interesting behavior for the meson decay constants in the axial sector as functions of the quark mass. Our approach was partially inspired by the holographic QCD model of [10], where chiral symmetry breaking maps to tachyon condensation in string theory. Our results agree qualitatively with [10] in the regime of large $m_{q}$ but differ in the chiral limit. The holographic model of [11] was another useful guide in our approach.

The paper is organized as follows. In Sec. II we briefly review chiral symmetry breaking in the hard wall and soft wall models. In Sec. III we present the nonlinear extensions of the soft wall model based on a Higgs potential. We perform a systematic analysis of the background including holographic renormalization for the chiral condensate. Solving the effective Schrödinger equations for the 5D field perturbations, we obtain the meson masses and decay constants. In Sec. IV we introduce a running mass for the 5D tachyonic field and investigate its effect on the chiral condensate, the meson spectrum, and decay constants. We present our conclusions in Sec. V. Appendix A describes the equations for the 5D field perturbations and the dictionary for the meson decay constants. Appendix B briefly reviews the holographic model of [11], while Appendix C describes some complementary numerical results.

\section{HARD AND SOFT WALL MODELS FOR CHIRAL SYMMETRY BREAKING}

Holographic models for QCD at zero temperature satisfy Poincaré invariance. The 5D metric for those models is usually written as

$$
d s^{2}=e^{2 A_{s}(z)}\left[-d t^{2}+d \vec{x}^{2}+d z^{2}\right],
$$

where $A_{s}(z)$ is the (string-frame) warp factor given as a function of the conformal coordinate $z$. In this work we are interested in the simplest backgrounds in which the 5D metric is just Lorentzian anti-de Sitter (AdS), i.e., $A_{s}(z)=-\ln z{ }^{1}$ Simplifying the background as much as possible allows us to focus on the dynamics of chiral symmetry breaking.

\section{A. The hard wall model}

In the hard wall model [6] the 5D background is given by

$$
d s^{2}=\frac{1}{z^{2}}\left[-d t^{2}+d \vec{x}^{2}+d z^{2}\right], \quad 0<z \leq z_{0},
$$

\footnotetext{
${ }^{1}$ As is usual in holographic QCD, we work in units in which the AdS radius is set to 1 .
} 
which corresponds to a 5D Lorentzian AdS space ending in an IR hard wall at $z=z_{0}$. A nice property of the hard wall model is that it satisfies the confinement criterion found in [2]. Soon it was realized that the hard wall model allows us to estimate the spectrum of glueballs [12].

The flavor sector of the hard wall model was introduced in $[4,5]$. The key ingredients were the 4D quark mass operator $\bar{q}_{R} q_{L}$ as well as the left and right $4 \mathrm{D}$ currents $J_{(L / R)}^{\mu, a}=\bar{q}_{L / R} \gamma^{\mu} T^{a} q_{L / R}$ associated with the chiral symmetry group $S U\left(N_{f}\right)_{L} \times S U\left(N_{f}\right)_{R}$. According to the AdS/CFT dictionary, the 4D quark mass operator maps to a $5 \mathrm{D}$ scalar $X$ field with mass given by the relation $m_{X}^{2}=\Delta(\Delta-4)$, with $\Delta$ the conformal dimension of the quark mass operator. In the extreme ultraviolet (UV) we have $\Delta=3$ and this corresponds to $m_{X}^{2}=-3$. This $5 \mathrm{D}$ mass would indicate an instability in Minkowski space, and therefore the 5D field $X$ is usually called the tachyon. The left and right 4D currents, on the other hand, are conserved in the extreme UV, so they are mapped to $5 \mathrm{D}$ gauge fields $A_{L / R}^{\mu, a}$.

In QCD chiral symmetry is broken dynamically in the IR due to a nontrivial vacuum and the dynamics of the quark mass operator. An important quantity is the so-called chiral condensate $\sigma$, which is the VEV of the quark mass operator, i.e., $\sigma=\langle\bar{q} q\rangle$. The authors of [4,5] realized that chiral symmetry breaking in $4 \mathrm{D}$ corresponds to $5 \mathrm{D}$ gauge symmetry breaking of the fields $A_{L / R}^{\mu, a}$ driven by a nontrivial scalar field $X$. In this work we use the conventions of [4] and write the $5 \mathrm{D}$ action as

$$
\begin{aligned}
S= & -\int d^{5} x \sqrt{-g} \operatorname{Tr}\left[\left|D_{m} X\right|^{2}+m_{X}^{2}|X|^{2}\right. \\
& \left.+\frac{1}{g_{5}^{2}} F_{m n}^{(L) 2}+\frac{1}{4 g_{5}^{2}} F_{m n}^{(R) 2}\right]
\end{aligned}
$$

where

$$
\begin{aligned}
F_{m n}^{(L / R)} & =\partial_{m} A_{n}^{(L / R)}-\partial_{n} A_{m}^{(L / R)}-i\left[A_{m}^{(L / R)}, A_{n}^{(L / R)}\right] \\
D_{m} X & =\partial_{m} X-i A_{m}^{(L)} X+i X A_{m}^{(R)} .
\end{aligned}
$$

Note that under the non-Abelian gauge group $S U\left(N_{f}\right)_{L} \times$ $S U\left(N_{f}\right)_{R}$ the left and right vector fields $A_{m}^{(L / R)}$ transform as adjoint fields, whereas the scalar field $X$ behaves as a bifundamental. From now on we take $N_{f}=2$ and make the assumption of isospin (flavor) symmetry, i.e., $m_{u}=m_{d}$ and $\sigma_{u}=\sigma_{d}$, which is a good approximation for the light quark sector in QCD. We therefore take the following ansatz for the 5D tachyonic field:

$$
X(z)=\frac{1}{2} v(z) I_{2 \times 2}
$$

In QCD at zero temperature (and zero density) we do not expect any vectorial condensate, so we take the following ansatz for the 5D gauge fields:

$$
A_{m}^{L / R}=0
$$

The 5D action in Eq. (3) reduces to a one-dimensional (1D) action for the field $v(z)$, and the Euler-Lagrange equation takes the form

$$
\left[\left(z \partial_{z}\right)^{2}-4 z \partial_{z}-m_{X}^{2}\right] v=0
$$

with the exact solution

$$
v(z)=c_{1} z^{\Delta_{-}}+c_{3} z^{\Delta_{+}}, \quad \Delta_{ \pm}=2 \pm \sqrt{4+m_{X}^{2}} .
$$

This has the expected scaling behavior for a 5D scalar field dual to an operator of dimension $\Delta_{+}$and a coupling of conformal dimension $\Delta_{-}=4-\Delta_{+}$. In order to match to the quark mass operator in the extreme UV, we choose $\Delta_{+}=3$. The source coefficient $c_{1}$ is related to the quark mass $m_{q}$ by $c_{1}=m_{q} \zeta$, where $\zeta$ is a normalization constant. This constant is usually fixed as $\zeta=\sqrt{N_{c}} / 2 \pi$ to be consistent with counting rules of large- $N_{c}$ QCD [13]. The VEV coefficient $c_{3}$ will be related to the quark condensate $\sigma_{u}$. The precise relation is scheme dependent and will be obtained in the next section.

In the hard wall model the VEV coefficient $c_{3}$ is fixed by boundary conditions at the IR wall $z=z_{0}$. This differs from what we expect in QCD, where the chiral condensate is generated dynamically due to a nontrivial vacuum. The hard wall model, however, has the nice feature of being the simplest model that describes at the same time confinement and chiral symmetry breaking.

\section{B. The (linear) soft wall model}

A shortcoming of the hard wall model is that it leads to hadronic masses that grow too fast as we increase the radial number. For example, the resonances of the $\rho$ meson have squared masses that grow as $m_{\rho^{(n)}}^{2} \sim n^{2}$ for large $n$. Experimental data, on the other hand, indicates an approximate linear dependence for the squared masses, i.e., $m_{\rho^{(n)}}^{2} \sim n$.

The quadratic dependence in the hard wall model can be thought of as a consequence of having a metric that abruptly ends at a cutoff $z=z_{0}$. Motivated by this problem, Karch et al. [7] proposed the idea of a smooth cutoff driven by a background scalar field $\Phi(z)$ so that the geometry does not abruptly end. This was inspired by string theory, where the field $\Phi$ is known as the dilaton. Later works have explored the backreaction of this background field on the 
AdS metric and how it maps to the Yang-Mills operator $\operatorname{Tr} F^{2}[14-16] .^{2}$

In the soft wall (SW) model the background is given by

$$
d s^{2}=\frac{1}{z^{2}}\left[-d t^{2}+d \vec{x}^{2}+d z^{2}\right], \quad \Phi(z)=\phi_{\infty} z^{2},
$$

where $\phi_{\infty}$ is a constant providing an IR mass scale. The flavor sector of the soft wall model is described by the action

$$
\begin{aligned}
S= & -\int d^{5} x \sqrt{-g} e^{-\Phi(z)} \operatorname{Tr}\left[\left|D_{m} X\right|^{2}+m_{X}^{2}|X|^{2}\right. \\
& \left.+\frac{1}{g_{5}^{2}} F_{m n}^{(L) 2}+\frac{1}{4 g_{5}^{2}} F_{m n}^{(R) 2}\right] .
\end{aligned}
$$

The positive quadratic $z$ dependence of $\Phi$ leads, at large $z$, to a harmonic oscillator form for the effective Schrödinger potentials for the field perturbations. This guarantees a linear dependence on the radial number for the meson squared masses, i.e., $m_{(n)}^{2} \sim n$.

In the flavor sector we take again the ansatz (5) and (6) for the background fields, and this time we obtain the equation

$$
\left[z^{2} \partial_{z}^{2}-\left(3+2 \phi_{\infty} z^{2}\right) z \partial_{z}-m_{X}^{2}\right] v=0
$$

In order to find an exact solution, we rewrite Eq. (11) in terms of a new variable $x \equiv \phi_{\infty} z^{2}$ and redefine the field $v(x)$ as $x^{\beta} \tilde{v}(x)$. We arrive at the second order differential equation

$$
\left\{x^{2} \partial_{x}^{2}+[2 \beta-1-x] x \partial_{x}+\beta^{2}-2 \beta-\frac{m_{X}^{2}}{4}-\beta x\right\} \tilde{v}=0 .
$$

This equation becomes Kummer's equation if

$$
\beta^{2}-2 \beta-\frac{m_{X}^{2}}{4}=0 \quad \rightarrow \quad \beta=1 \pm \sqrt{1+\frac{m_{X}^{2}}{4}}=\frac{\Delta_{ \pm}}{2} .
$$

The solutions for $\tilde{v}(z)$ are of the form $M(\beta, 2 \beta-1 ; x)$ (Kummer) and $U(\beta, 2 \beta-1 ; x)$ (Tricomi). From this analysis we conclude that the exact solution for $v(z)$ can be written as

\footnotetext{
${ }^{2}$ The AdS deformation by a scalar field may also be interpreted in terms of supersymmetry breaking; see, e.g., [17].
}

$$
\begin{aligned}
v(z)= & \tilde{c}_{1} z^{\Delta_{-}} U\left(\frac{\Delta_{-}}{2}, \Delta_{-}-1 ; \phi_{\infty} z^{2}\right) \\
& +\tilde{c}_{3} z^{\Delta_{+}} M\left(\frac{\Delta_{+}}{2}, \Delta_{+}-1 ; \phi_{\infty} z^{2}\right),
\end{aligned}
$$

where $\tilde{c}_{1}$ and $\tilde{c}_{3}$ are constant coefficients. For $m_{X}^{2}=-3$ we have $\Delta_{-}=1$ and $\Delta_{+}=3$. This exact solution was first obtained in [18]. In the limit $x \rightarrow \infty$ we have $M(a, b ; x) \sim$ $e^{x} x^{a-b}$ and $U(a, b ; x) \sim x^{-a}$; therefore, we need to set $\tilde{c}_{3}=0$ in order to avoid a divergent solution in the IR. The tachyon solution in Eq. (14) becomes

$$
v(z)=\frac{\sqrt{\pi}}{2} c_{1} z U\left(\frac{1}{2}, 0 ; \phi_{\infty} z^{2}\right)
$$

where $\tilde{c}_{1}$ was rewritten as $(\sqrt{\pi} / 2) c_{1}$. In the UV (small $z$ ), the solution in Eq. (15) behaves as

$$
v^{\mathrm{UV}}(z)=c_{1} z+d_{3}\left(c_{1}\right) z^{3} \ln z+c_{3}\left(c_{1}\right) z^{3}+\ldots .
$$

The logarithmic term could have been anticipated from an asymptotic analysis using the Frobenius method. Coefficients $d_{3}$ and $c_{3}$ are both actually proportional to $c_{1}$ and then vanish in the chiral limit $c_{1} \rightarrow 0$. In the IR the tachyon solution (15) behaves as

$$
v^{\mathrm{IR}}(z)=C_{0}\left[1+\mathcal{O}\left(z^{-2}\right)\right], \quad C_{0}=\frac{\sqrt{\pi}}{2} \frac{c_{1}}{\sqrt{\phi_{\infty}}} .
$$

The parameter $C_{0}$ characterizes the tachyon solution at large $z$. As expected, the solution is regular in the IR. This regular solution was, however, chosen by setting $\tilde{c}_{3}=0$. In the next section we will introduce a nonlinear potential for the tachyonic field. The nonlinearity of the new differential equation will naturally lead to regular solutions in the IR without the need of fixing any integration constant.

The soft wall model represents an important step toward the construction of a minimal model for describing the flavor sector in holographic QCD. The positive quadratic behavior of the dilaton $\Phi(z)$ leads naturally to a linear spectrum for the mesons. Moreover, when taking backreaction into account, the same positive quadratic dilaton leads to a linear spectrum for glueballs $[15,16]$. The soft wall model has also served as a useful guide to other approaches, such as light-front holography [19].

\section{NONLINEAR SOFT WALL MODELS}

Karch et al. [7] realized that the linear dependence of $c_{3}$ on $c_{1}$ differed significantly from what one would expect in QCD for the chiral condensate $\sigma_{q}$ as a function of the quark mass $m_{q}$. They suggested that the model could be improved by adding nonlinear terms to the tachyonic potential. The nonlinearity would lead to solutions in the IR characterized by only one parameter, $C_{0}$, and the relation between the IR 
parameter $C_{0}$ and the $\mathrm{UV}$ parameter $c_{1}$ would also be nonlinear.

In this work we investigate a nonlinear potential of the Higgs form

$$
U(|X|)=m_{X}^{2}|X|^{2}+\lambda|X|^{4} .
$$

This time the 5D action for the flavor sector becomes

$$
\begin{aligned}
S= & -\int d^{5} x \sqrt{-g} e^{-\Phi(z)} \operatorname{Tr}\left[\left|D_{m} X\right|^{2}+m_{X}^{2}|X|^{2}+\lambda|X|^{4}\right. \\
& \left.+\frac{1}{g_{5}^{2}} F_{m n}^{(L) 2}+\frac{1}{4 g_{5}^{2}} F_{m n}^{(R) 2}\right] .
\end{aligned}
$$

We will consider both cases $\lambda<0$ and $\lambda>0$ and also recover the original soft wall model in the limit $\lambda \rightarrow 0$. As in the previous cases, we take $m_{X}^{2}=-3$ for the tachyonic field.

For the background fields we take again the ansatz (5) and (6), and this time we obtain a nonlinear equation for the tachyon:

$$
\left[z^{2} \partial_{z}^{2}-\left(3+2 \phi_{\infty} z^{2}\right) z \partial_{z}+3\right] v-\frac{\lambda}{2} v^{3}=0 .
$$

Because of the nonlinearity of the differential equation, we cannot obtain an analytic solution. In the next subsections we describe the asymptotic solutions for the tachyon in the UV and IR regimes and present our numerical results for the tachyon profiles. Then we investigate the meson spectrum for the cases $\lambda<0$ and $\lambda>0$. We finish the section by describing the meson decay constants.

\section{A. Asymptotic analysis}

In the UV we consider the Frobenius ansatz

$v(z)=c_{1} z+d_{3} z^{3} \ln z+c_{3} z^{3}+d_{5} z^{5} \ln z+c_{5} z^{5}+\ldots$

Plugging this ansatz into the tachyon equation (20), we find the UV coefficients

$d_{3}=\frac{1}{4} c_{1}\left(c_{1}^{2} \lambda+4 \phi_{\infty}\right), \quad d_{5}=\frac{3}{64} c_{1}\left(c_{1}^{2} \lambda+4 \phi_{\infty}\right)^{2}+$,

$c_{5}=\frac{1}{256}\left(c_{1}^{2} \lambda+4 \phi_{\infty}\right)\left(-9 c_{1}^{3} \lambda-20 c_{1} \phi_{\infty}+48 c_{3}\right) \ldots$.

Note that $d_{3}$ and $d_{5}$ depend only on $c_{1}$, whereas $c_{5}$ depends on $c_{1}$ and $c_{3}$. In the special case $c_{1}^{2} \lambda+4 \phi_{\infty}=0$ all of the subleading terms vanish and the linear solution $v(z)=c_{1} z$ becomes exact. This is possible only for $\lambda<0$ because in the soft wall model we have $\phi_{\infty}>0$.
The VEV parameter $c_{3}$ appears to be independent of $c_{1}$. We will see, however, that in the IR the tachyon solution is characterized by a single parameter $C_{0}$. The UV parameters $c_{1}$ and $c_{3}$ then can be thought of as functions of the IR parameter $C_{0}$. As a consequence, $c_{3}$ will depend on $c_{1}$ in a nonlinear fashion, as predicted in [7]. We remind the reader that the UV parameters $c_{1}$ and $c_{3}$ are related to the quark mass $m_{q}$ and chiral condensate $\sigma_{q}$, respectively. As far as we are concerned, the first nonlinear realization of chiral symmetry breaking via tachyon dynamics in terms of $c_{3}$ as a function of $c_{1}$ was developed in the holographic QCD model of [10]. ${ }^{3}$ More sophisticated models that take into account the backreaction of the tachyon were developed in [22-25].

In the IR it is convenient to work with the variable $y=1 / z$. The differential equation (20) may be written as

$$
\left[\left(y \partial_{y}\right)^{2}+2\left(2+\phi_{\infty} y^{-2}\right)\left(y \partial_{y}\right)+3\right] v-\frac{\lambda}{2} v^{3}=0 .
$$

We first consider the power ansatz

$$
v^{\mathrm{IR}}(y)=C_{0} y^{\alpha} .
$$

Plugging this ansatz into Eq. III A, we obtain the polynomial equation

$C_{0} y^{\alpha}\left(\alpha^{2}+4 \alpha+3\right)+C_{0} y^{\alpha-2}\left(2 \alpha \phi_{\infty}\right)-\frac{\lambda}{2} C_{0}^{3} y^{3 \alpha}=0$.

The first term in Eq. (25) is subleading compared to the second. The third term competes with the second term only when $\alpha<0$. We distinguish among three cases: $\alpha>-1$, $\alpha=-1$, and $\alpha<-1$. The last case is trivial because it leads to $C_{0}=0$.

In the case $\alpha>-1$ the second term always dominates and we find $\alpha=0$. This is the regular solution we are looking for and it admits the following expansion,

$$
v^{\mathrm{IR}}(y)=C_{0}+C_{2} y^{2}+C_{4} y^{4}+\ldots,
$$

with the subleading IR coefficients given by

$$
\begin{aligned}
& C_{2}=\frac{C_{0}}{8 \phi_{\infty}}\left(C_{0}^{2} \lambda-6\right), \\
& C_{4}=\frac{3 C_{0}}{128 \phi_{\infty}^{2}}\left(C_{0}^{2} \lambda-6\right)\left(C_{0}^{2} \lambda-10\right) .
\end{aligned}
$$

To guarantee the convergence of the series (26), we need a condition of the form $\left|C_{0}^{2} \lambda\right| / \phi_{\infty}<1$. In the special case $C_{0}^{2} \lambda-6=0$ all subleading coefficients vanish and the

\footnotetext{
${ }^{3}$ For earlier works on the dictionary between tachyon dynamics and the chiral condensate from a top-down approach, see $[20,21]$.
} 


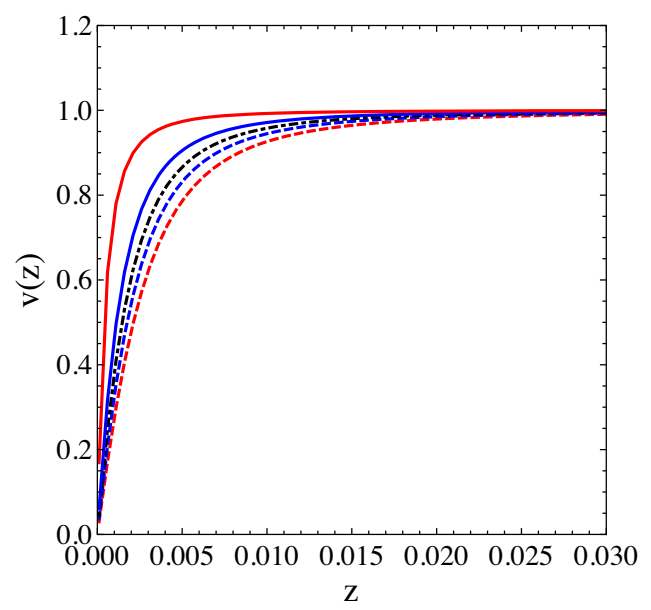

FIG. 1. Tachyon profiles for $C_{0}=1$ and $\lambda$ varying from -5 (red dashed line) to 5 (red solid line). The black dot-dashed profile in the middle corresponds to $\lambda=0$ (linear soft wall model).

constant solution is exact. This can be obtained only in the case $\lambda>0$ and, interestingly, it corresponds to the minimum of the Higgs potential $\partial U / \partial v=0$.

In the special case $\alpha=-1$ we see from Eq. (25) that the first term vanishes, whereas the second and third terms are of order $y^{-3}$, leading to the condition $C_{0}^{2} \lambda+4 \phi_{\infty}=0$. This divergent solution is linear, i.e., $v(z)=C_{0} z$, and it is valid only for $\lambda<0$. This linear solution appears to be exact, and it may be related to previous approaches to nonlinear soft wall (NLSW) models such as [11]. The model of [11] is briefly discussed in Appendix B.

\section{B. Numerical solution}

Once the asymptotic analysis has been done, we proceed to numerically solve the nonlinear differential equation for the tachyon field (20). We may integrate Eq. (20) from the UV to the IR using the UV asymptotic solution (21) to extract initial conditions. By matching the numerical solution to the IR analytic solution (26), we find a relation between the UV parameters $c_{1}$ and $c_{3}$ as well as the corresponding IR parameter $C_{0}$. Alternatively, we can integrate numerically Eq. (20) from the IR to the UV using the IR analytic solution (26), and this time the matching procedure allows us to extract the UV parameters $c_{1}$ and $c_{3}$ in terms of the IR parameter $C_{0}$.

We show in Fig. 1 typical profiles for the tachyon field $v(z)$ for fixed $C_{0}$ and different values of $\lambda$. The linear soft wall model corresponding to $\lambda=0$ is depicted by the black dot-dashed line, and we see that the effect of a nonlinear negative (positive) quartic coupling $\lambda$ is to deform the tachyon profile to the right (left). In the case of $\lambda>0$ we have an upper bound $C_{0}^{2} \lambda=6$ for the tachyon solution. As we approach that bound, the tachyon profile becomes constant in $z$, which is consistent with the asymptotic analysis done in the previous subsection.
Our numerical results for the UV parameters $c_{1}$ and $c_{3}$ as functions of the IR parameter $C_{0}$ are displayed in Fig. 2 for different values of $\lambda$. The linear soft wall model $(\lambda=0)$ is represented by the black dot-dashed line. All solutions enjoy the symmetry $\left(c_{1}, c_{3}\right) \leftrightarrow\left(-c_{1},-c_{3}\right)$ corresponding to the symmetry $v \leftrightarrow-v$ present in the differential equation (20). The physical regime, of course, corresponds to $c_{1}>0$. We see that the nonlinearity of the tachyon differential equation leads to nonlinear relations $c_{1}\left(C_{0}\right)$ and $c_{3}\left(C_{0}\right)$, as expected. However, in the chiral limit (corresponding to $c_{1} \rightarrow 0$ ) all parameters go to zero. In particular, the VEV parameter $c_{3}$ (associated with the 4D chiral condensate) vanishes. This differs significantly from QCD, where chiral symmetry is spontaneously broken in the chiral limit and therefore the chiral condensate is nonvanishing. Note that nonlinearity of the tachyon differential equation also brings saturation effects. In the case $\lambda>0$ there is an upper bound in $C_{0}$ (described in the previous paragraph), whereas in the case $\lambda<0$ we have an upper bound in $c_{1}$. An upper bound in $c_{1}$ implies a cutoff for the quark mass, which is not expected in QCD.

In Fig. 3 we show the VEV parameter $c_{3}$ as a function of the source parameter $c_{1}$ in the physical regime. This result will be interpreted in terms of the 4D chiral condensate $\langle\bar{q} q\rangle$ as a function of the quark mass $m_{q}$, as described in the next subsection. The linear relation $c_{3} \sim c_{1}$, characteristic of the linear soft wall model, is actually a good approximation at small $c_{1}$. For positive $\lambda$ the VEV parameter $c_{3}$ grows monotonically with $c_{1}$, which is the expected behavior for $\langle\bar{q} q\rangle$. A similar behavior was obtained in the holographic QCD model developed in [10]. The case of negative $\lambda$ leads to a nonmonotonic function $c_{3}\left(c_{1}\right)$. In that case the VEV parameter $c_{3}$ vanishes when $c_{1}$ reaches its upper bound. We conclude that the case $\lambda>0$ leads to the more realistic scenario. This could have been anticipated due to the fact that $\lambda>0$ in Eq. (18) corresponds to the Mexican hat potential for the Higgs field.

\section{Holographic renormalization and the chiral condensate}

In this subsection we describe the procedure of holographic renormalization for the nonlinear soft wall models proposed in this work. The case at hand is similar to the case of probe branes in a fixed background; see, e.g., $[10,26]$. The starting point is the on-shell action. For the background tachyonic field the action in Eq. (19), when taken on shell, can be written as

$$
S_{\mathrm{os}}=S_{\mathrm{Bdy}}+S_{\text {Int }} \text {, }
$$

where 

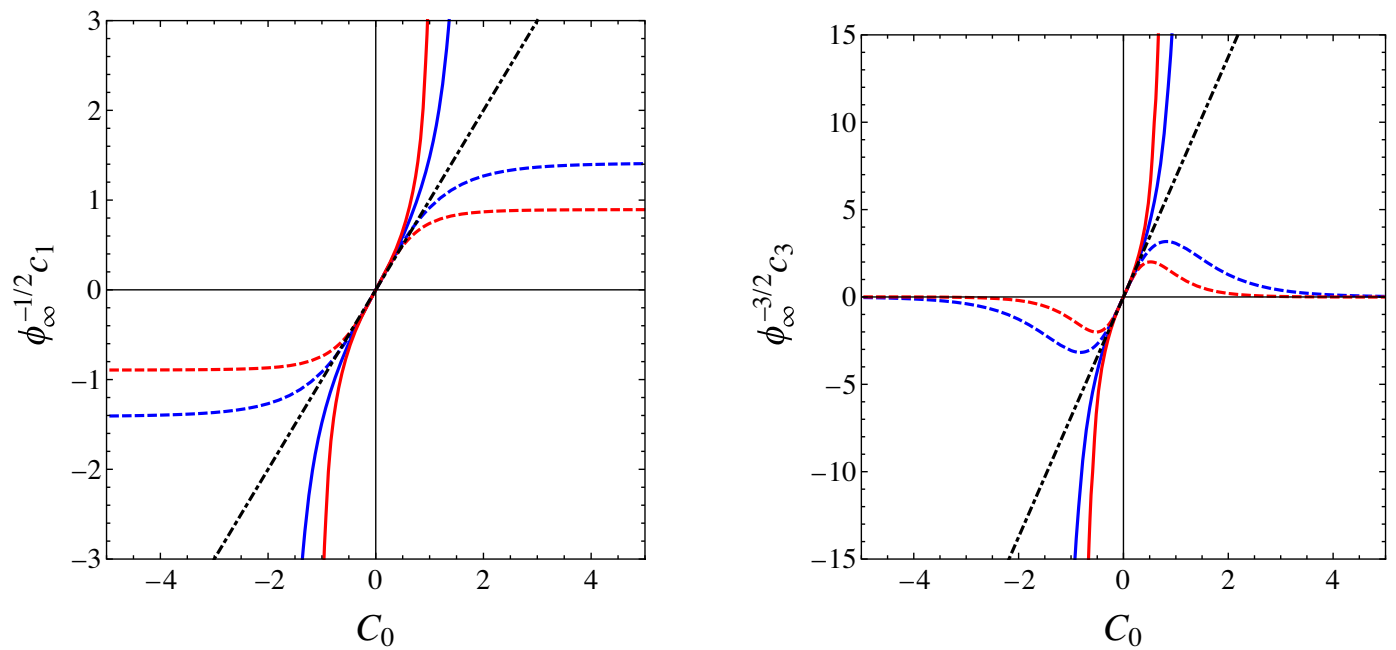

FIG. 2. Numerical results for (left panel) $c_{1}$ and (right panel) $c_{3}$ as functions of $C_{0}$ in units of $\sqrt{\phi_{\infty}}$. The blue and red solid lines (dashed lines) correspond to $\lambda=2$ and $\lambda=5(\lambda=-2$ and $\lambda=-5)$. The black dot-dashed line corresponds to $\lambda=0$ (linear soft wall model).

$S_{\mathrm{Bdy}}=-\frac{1}{2} \int d^{4} x \Pi_{z}\left(z_{0}\right) v\left(z_{0}\right), \quad \Pi_{z}=-z^{-3} e^{-\Phi(z)} \partial_{z} v$

$$
S_{\text {Int }}=\frac{\lambda}{8} \int d^{4} x d z z^{-5} e^{-\Phi(z)} v^{4}
$$

$S_{\text {Bdy }}$ is a boundary term (the AdS boundary is located at $z=z_{0}$ with $\left.z_{0} \rightarrow 0\right)$, and $\Pi_{z}=\partial \mathcal{L} / \partial\left(\partial_{z} v\right)$ is the conjugate momentum in $z$. The bulk term $S_{\text {Int }}$ appears because of the nonlinear term in the tachyon potential.

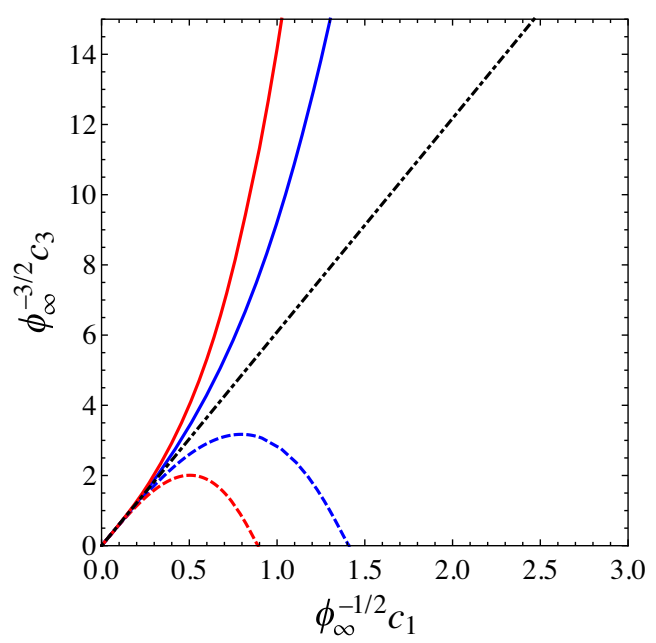

FIG. 3. VEV parameter $c_{3}$ as a function of the source parameter $c_{1}$, in units of $\sqrt{\phi_{\infty}}$, for different values of $\lambda$. The blue and red solid lines (dashed lines) correspond to $\lambda=2$ and $\lambda=5(\lambda=-2$ and $\lambda=-5)$. The black dot-dashed line corresponds to $\lambda=0$ (linear soft wall model).

\section{Counterterms and covariant subtraction}

Plugging the UV asymptotic solution (21) into the surface term (29), we see that it splits into divergent and finite pieces

$$
\begin{gathered}
S_{\mathrm{Bdy}}=S_{\mathrm{Bdy}}^{\mathrm{Div}}+S_{\mathrm{Bdy}}^{\mathrm{Fin}}, \\
S_{\mathrm{Bdy}}^{\mathrm{Div}}=\frac{1}{2} \int d^{4} x\left[c_{1}^{2} z_{0}^{-2}+c_{1}^{2}\left(c_{1}^{2} \lambda+4 \phi_{\infty}\right) \ln z_{0}\right], \\
S_{\mathrm{Bdy}}^{\mathrm{Fin}}=\frac{1}{2} \int d^{4} x\left[\frac{1}{4} c_{1}^{4} \lambda+4 c_{1} c_{3}\right] .
\end{gathered}
$$

We have omitted in Eq. (33) the terms that vanish in the limit $z_{0} \rightarrow 0$. The bulk term (30) can not be split in a simple way, but, from Eq. (21), we find the divergent piece

$$
S_{\text {Int }}^{\text {Div }}=-\frac{\lambda}{8} \int d^{4} x c_{1}^{2} \ln z_{0}
$$

and simply define the finite piece as

$$
S_{\text {Int }}^{\text {Fin }}=S_{\text {Int }}-S_{\text {Int }}^{\text {Div }} .
$$

In order to cancel the UV divergences (32)-(34) in a consistent way, we introduce the covariant counterterms

$$
\begin{aligned}
S_{\mathrm{ct}}= & -\int d^{4} x \sqrt{-\gamma}\left\{a_{1} v^{2}\left(z_{0}\right)\right. \\
& +\ln z_{0}\left[a_{2} \Phi\left(z_{0}\right) v^{2}\left(z_{0}\right)-a_{3} \lambda v^{4}\left(z_{0}\right)\right] \\
& \left.+a_{4} \Phi\left(z_{0}\right) v^{2}\left(z_{0}\right)-a_{5} \lambda v^{4}\left(z_{0}\right)\right\} .
\end{aligned}
$$

The first three terms in Eq. (36) are the minimum required to cancel the UV divergences. The last two terms in 
Eq. (36) are finite counterterms associated with the renormalization scheme dependence of the 4D dual theory. The counterterms action in Eq. (36) can be split into divergent and finite pieces:

$$
S_{\mathrm{ct}}=S_{\mathrm{ct}}^{\mathrm{Div}}+S_{\mathrm{ct}}^{\text {Fin }},
$$

$$
\begin{aligned}
S_{\mathrm{ct}}^{\mathrm{Div}}= & -\int d^{4} x\left\{a_{1} c_{1}^{2} z_{0}^{-2}\right. \\
+ & {\left.\left[\left(2 a_{1}+a_{2}\right) c_{1}^{2} \phi_{\infty}+\left(\frac{a_{1}}{2}-a_{3}\right) c_{1}^{4} \lambda\right] \ln z_{0}\right\}, } \\
S_{\mathrm{ct}}^{\mathrm{Fin}} & =-\int d^{4} x\left[2 a_{1} c_{1} c_{3}+a_{4} c_{1}^{2} \phi_{\infty}-a_{5} c_{1}^{4} \lambda\right] .
\end{aligned}
$$

The renormalized action is then defined as

$$
S_{\text {Ren }}=S_{\text {Bdy }}+S_{\text {Int }}+S_{\mathrm{ct}} \text {. }
$$

The UV divergences cancel for

$$
a_{1}=\frac{1}{2}, \quad a_{2}=1, \quad a_{3}=-\frac{1}{8},
$$

and the renormalized action takes the form

$$
S_{\mathrm{Ren}}=S_{\mathrm{Bdy}}^{\mathrm{Fin}}+S_{\mathrm{Int}}^{\mathrm{Fin}}+S_{\mathrm{ct}}^{\mathrm{Fin}} .
$$

\section{The chiral condensate and the renormalized Hamiltonian}

The conformal field theory (CFT) deformation due to the quark mass operator has the form $\int d^{4} x m_{q}\langle\bar{q} q\rangle$. The holographic dictionary for the chiral condensate then takes the form

$$
\langle\bar{q} q\rangle=\zeta\left[\frac{\delta S_{\mathrm{os}}}{\delta c_{1}}+\frac{\delta S_{\mathrm{ct}}}{\delta c_{1}}\right],
$$

where $\zeta=\sqrt{N_{c}} / 2 \pi$ is the normalization constant introduced in the previous section. We identify the first term,

$$
\begin{aligned}
\frac{\delta S_{\mathrm{os}}}{\delta c_{1}}= & -\frac{\partial v\left(z_{0}\right)}{c_{1}} \Pi_{z}\left(z_{0}\right) \\
= & c_{1} z_{0}^{-2}+\left(4 c_{1} \phi_{\infty}-\frac{3}{2} c_{1}^{3} \lambda\right) \ln z_{0} \\
& +3 c_{3}+c_{1} \partial_{c_{1}} c_{3}+\frac{1}{4} c_{1}^{3} \lambda,
\end{aligned}
$$

as the bare contribution and the second term,

$$
\begin{aligned}
\frac{\delta S_{\mathrm{ct}}}{\delta c_{1}}= & -c_{1} z_{0}^{-2}-\left(4 c_{1} \phi_{\infty}+\frac{3}{2} c_{1}^{3} \lambda\right) \ln z_{0}-c_{3}-c_{1} \partial_{c_{1}} c_{3} \\
& -2 a_{4} c_{1} \phi_{\infty}+4 a_{5} c_{1}^{3} \lambda,
\end{aligned}
$$

as the counterterm contribution. From Eqs. (43)-(45) we see that the UV divergences cancel, and we arrive at the final expression for the chiral condensate:

$$
\langle\bar{q} q\rangle=\zeta\left[2 c_{3}-2 a_{4} c_{1} \phi_{\infty}+\frac{1}{4} c_{1}^{3} \lambda\left(1+16 a_{5}\right)\right] .
$$

The coefficients $a_{4}$ and $a_{5}$ reflect the scheme dependence of the chiral condensate. Since we already know that $c_{3}$ has a very similar behavior to the QCD chiral condensate, we suspect that the natural choice for fixing the scheme would be choosing $a_{4}=0$ and $a_{5}=-1 / 16$. In Fig. 4 we plot the

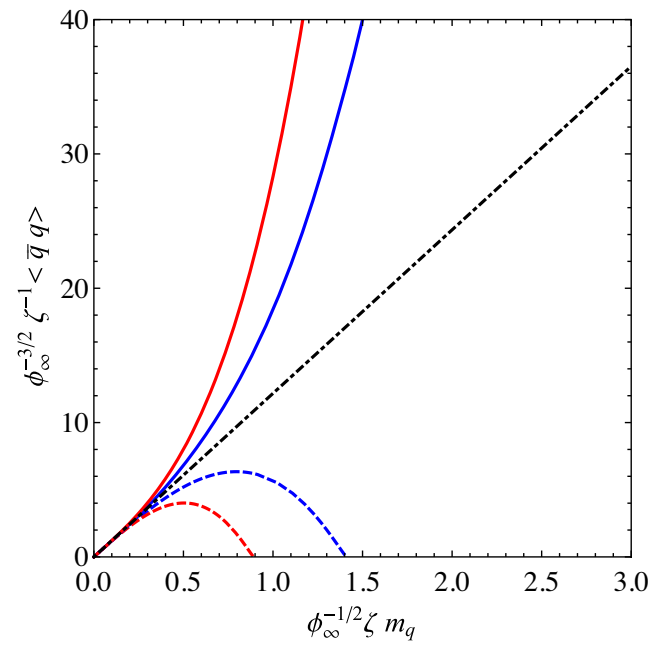

FIG. 4. [Left (right) panel] The renormalized Hamiltonian (chiral condensate) as a function of the UV parameter $c_{1}$ (quark mass) in units of $\sqrt{\phi_{\infty}}$. The scheme was fixed by setting $a_{4}=0$ and $a_{5}=-1 / 16$. The blue and red solid (dashed) lines correspond to $\lambda=2$ and $\lambda=5(\lambda=-2$ and $\lambda=-5)$, respectively. The black dot-dashed line corresponds to $\lambda=0$ (linear case). 
renormalized Hamiltonian and the chiral condensate, in units of $\sqrt{\phi_{\infty}}$, for that particular scheme. As promised, we find a nonlinear relation between the chiral condensate and the quark mass. As described in the previous subsection, the model with $\lambda>0$ provides a more realistic description of the chiral condensate. This conclusion will be supported by the analysis of the meson spectrum performed in the next subsection. It can be checked that the chiral condensate could have been obtained directly using the relation $\langle\bar{q} q\rangle=-\partial H^{\text {Ren }} / \partial m_{q}$.

We finish this subsection with a general conclusion for the nonlinear soft wall models presented in this work. As described in the previous subsection, the VEV parameter $c_{3}$ always vanishes in the limit $c_{1} \rightarrow 0$. Regardless of the renormalization scheme, this result implies that the chiral condensate $\langle\bar{q} q\rangle$ always vanishes in the chiral limit, in sharp contrast to QCD. This means that the nonlinear models at hand never lead to spontaneous symmetry breaking. This result will be confirmed later when we conclude that pseudo-Nambu-Goldstone (NG) modes are absent in the spectrum of pseudoscalar mesons. An original solution to this problem was found in $[11,27]$, where the dilaton profile was modified drastically near the boundary to allow for a description of spontaneous symmetry breaking. The authors of $[11,27]$ realized that the dilaton profile had to be negative in the UV to guarantee spontaneous symmetry breaking. In this work we take a more conservative approach of a positive quadratic dilaton to avoid instabilities in the fluctuations associated with the meson spectrum. Nevertheless, the model in [11,27] deserves further systematic study. That model is briefly reviewed in Appendix B.

\section{Meson spectrum $(\lambda<\mathbf{0})$}

The non-Abelian Higgs action in Eq. (19) describes the dynamics of the scalar tachyonic field $X$ as well as the left and right gauge fields $A_{m}^{(L / R)}$. At the beginning of this section we analyzed the background tachyonic field $X=$ $\frac{1}{2} v(z)$ (the background gauge fields were set to zero). Now we consider the perturbations of the fields $X$ and $A_{m}^{(L / R)}$. As described in Appendix A, the perturbations associated with the tachyonic field are the scalar field $S$ and the pseudoscalar fields $\pi^{a}$. The former will describe a tower of scalar mesons, whereas the latter will be related to the pseudoscalar mesons (pions in the case of $N_{f}=2$ ). On the other hand, the gauge field fluctuations will be written as $A_{m}^{(L / R)}=V_{m} \pm A_{m}$, with $V_{m}=V_{m}^{a} T^{a}$ and $A_{m}=A_{m}^{a} T^{a}$ identified as the $5 \mathrm{D}$ fields dual to the vectorial and axial currents in the chiral symmetry group.

As described in Appendix A, the vector field $V_{m}^{a}$ decomposes as $\left(V_{z}^{a}, V_{\mu}^{a}\right)$, and $V_{\mu}^{a}$ will describe a tower of $4 \mathrm{D}$ vector mesons. The axial-vector field $A_{m}^{a}$ decomposes as $\left(A_{z}^{a}, A_{\mu}^{a}\right)$ and, in turn, $A_{\mu}^{a}$ decomposes into
TABLE I. The mass of the vector mesons (in $\mathrm{MeV}$ ) obtained in the soft wall model, compared against the holographic model [11] and experimental results from PDG [29].

\begin{tabular}{lccc}
\hline \hline$n$ & SW [7] & GKK [11] & $\rho$ experimental [29] \\
\hline 1 & 776 & 475 & $776 \pm 1$ \\
2 & 1097 & 1129 & $1282 \pm 37$ \\
3 & 1344 & 1429 & $1465 \pm 25$ \\
4 & 1552 & 1674 & $1720 \pm 20$ \\
5 & 1735 & 1884 & $1909 \pm 30$ \\
6 & 1901 & 2072 & $2149 \pm 17$ \\
7 & 2053 & 2243 & $2265 \pm 40$ \\
\hline \hline
\end{tabular}

transverse $A_{\mu}^{\perp, a}$ and longitudinal parts $\partial_{\mu} \phi^{a}$. The fields $A_{\mu}^{\perp, a}$ will describe a tower of axial-vector mesons, whereas the fields $\phi^{a}$ will couple to the fields $\pi^{a}$ and describe a tower of pseudoscalar mesons. The equations for the vector sector $V_{\mu}^{\perp, a}$, scalar sector $S$, axial-vector sector $A_{\mu}^{\perp, a}$, and pseudoscalar sector $\left(\pi^{a}, \phi^{a}\right)$ are obtained from a second order expansion of the action (19), as described in Appendix A.

\section{Spectrum of the vector sector}

We start with the equation of motion (A9) (the flavor index is hidden in the following analysis). After performing the Fourier transform $V_{\mu}\left(x^{\mu}, z\right) \rightarrow V_{\mu}\left(k^{\mu}, z\right)$ on Eq. (A9), where we have set $\square \rightarrow m_{V}^{2}$, the equation may be written in the Schrödinger form through the transformation $V_{\mu}=\xi_{\mu} e^{-B_{V}} \psi_{v_{n}}$, where $B_{V}=\left(A_{s}-\Phi\right) / 2$ and $\xi_{\mu}$ is a (transverse) polarization vector. ${ }^{4}$ The effective Schrödinger equation reads

$$
\left[-\partial_{z}^{2}+V_{V}\right] \psi_{v_{n}}=m_{V}^{2} \psi_{v_{n}},
$$

where the potential is given by

$$
V_{V}=\left(\partial_{z} B_{V}\right)^{2}+\partial_{z}^{2} B_{V}
$$

In this case the problem has an exact solution [7]:

$$
m_{V}^{2}=4 \phi_{\infty}(1+n), \quad n=0,1,2, \ldots
$$

At this point the free parameter is $\phi_{\infty}$, and we may fix the value of this parameter by comparing the first vector state with the corresponding experimental value of the $\rho$ meson, as was done in, e.g., [28]. We obtain the value $\phi_{\infty}=(388 \mathrm{MeV})^{2}$.

The spectrum obtained is shown in Table I, labeled as SW, and compared against the holographic model of [11] and experimental data [29]. The details of [11] are given in Appendix B.

\footnotetext{
${ }^{4} \mathrm{We}$ are introducing again the string-frame warp factor $A_{s}=-\ln z$.
} 


\section{Spectrum of the scalar sector}

Now we proceed to calculate the spectrum of the scalar mesons. This sector is obtained from the fluctuations of the tachyon field, see Eq. (A1), where $S(x, z)$ represents the scalar field related to the scalar mesons. After performing the Fourier transform $S\left(x^{\mu}, z\right) \rightarrow S\left(k^{\mu}, z\right)$ on Eq. (A11), where we set $\square \rightarrow m_{s}^{2}$, we arrive at the following equation:

$$
\begin{aligned}
& e^{-3 A_{s}+\Phi} \partial_{z}\left(e^{3 A_{s}-\Phi} \partial_{z} S(k, z)\right)+m_{s}^{2} S(k, z) \\
& -e^{2 A_{s}}\left(m_{X}^{2}(z)+\frac{3}{2} \lambda v^{2}(z)\right) S(k, z)=0,
\end{aligned}
$$

where $m_{X}^{2}(z)=-3$. We rewrite the last equation in a Schrödinger form, redefining the scalar modes as $S_{n}=$ $e^{-B_{S}} \psi_{s_{n}}(z)$, where $B_{S}=3 A_{s} / 2-\Phi / 2$. Thus, we get

$$
-\partial_{z}^{2} \psi_{s_{n}}+V_{S} \psi_{s_{n}}=m_{s}^{2} \psi_{s_{n}}
$$

with the Schrödinger potential given by

$$
V_{S}=\left(\partial_{z} B_{S}\right)^{2}+\partial_{z}^{2} B_{S}+e^{2 A_{s}}\left(m_{X}^{2}+\frac{3 \lambda}{2} v^{2}(z)\right)
$$

For $\lambda=0$ the potential (52) reduces to the one obtained in [18]. Notice how the parameter $\lambda$ controls the minimum value of the potential (52), as shown in Fig. 5. As we increase $\lambda$ the minimum increases, and hence also the masses of the scalar mesons. For $\lambda<0$ the potential allows us to describe very light states. This statement is supported by the results displayed in Fig. 6, where we can see the evolution of the meson with the parameter $C_{0}$ (left panel) and $c_{1}$ (right panel). Those results were obtained for $\lambda=-2$ (solid lines) and $\lambda=0$ (dashed lines).

Those results indicate the possibility of fixing the parameter $C_{0}$ for a given $\lambda$, requiring the first eigenvalue of the Schrödinger equation to match the mass of the scalar meson $f_{0}(550 \mathrm{MeV}) .^{5}$ However, the status of the $f_{0}(550 \mathrm{MeV})$ as a scalar meson is not established [29]. We follow a more conservative approach and consider $f_{0}(980 \mathrm{MeV})$ as the first scalar meson, as in [32]. As the upper limit for the scalar mass in our model is the one obtained for $\lambda=0, m_{s}=950 \mathrm{MeV}$ (see Fig. 6), it is not possible to reach the state $f_{0}(980 \mathrm{MeV})$ when $\lambda<0$.

It is worth mentioning that the behavior of the scalar meson mass as a function of the quark mass, i.e., $c_{1}$, displayed in Fig. 6 is the opposite of that expected in QCD. Hence, the model with $\lambda<0$ is pathological in the scalar sector.

\footnotetext{
${ }^{5}$ There were some attempts to obtain a light scalar meson in the top-down approach; see, e.g., [30,31].
}

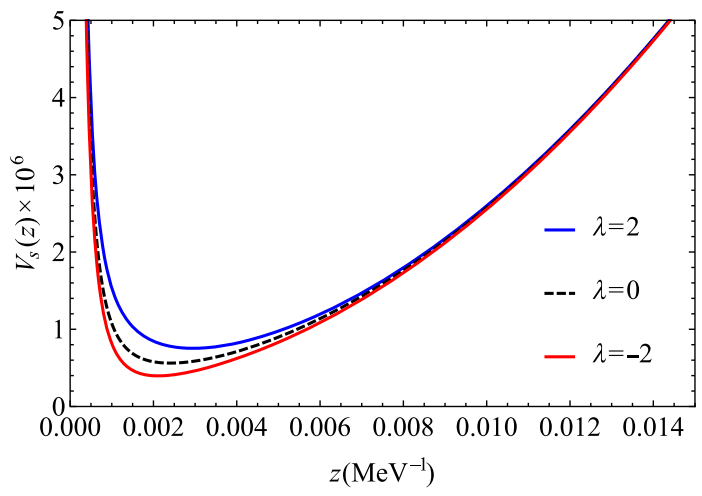

FIG. 5. Potential of the Schrödinger equation for $\phi_{\infty}=$ $(388 \mathrm{MeV})^{2}$ and three different values of the parameter $\lambda$.

\section{Spectrum of the axial-vector sector}

After performing the Fourier transform $A_{\perp}^{\mu}\left(x^{\mu}, z\right) \rightarrow$ $A_{\perp}^{\mu}\left(k^{\mu}, z\right)$ on Eq. (A13), with $\square \rightarrow m_{A}^{2}$, redefining the axial-vector mode as $A_{\mu}=\xi_{\mu} e^{-B_{A}} \psi_{a_{n}}$, where $B_{A}=$ $\left(A_{s}-\Phi\right) / 2$, we arrive at the Schrödinger equation

$$
-\partial_{z}^{2} \psi_{a_{n}}+V_{A} \psi_{a_{n}}=m_{A}^{2} \psi_{a_{n}}
$$

with the Schrödinger potential given by

$$
V_{A}=\left(\partial_{z} B_{A}\right)^{2}+\partial_{z}^{2} B_{A}+g_{5}^{2} e^{2 A_{s}} v^{2}(z) .
$$

The left panel of Fig. 7 shows our results for the masses of the axial-vector states as a function of the parameter $C_{0}$, compared against the corresponding values in the linear soft wall model, i.e., $\lambda=0$. The right panel of the figure shows how the masses depend on the parameter of the quark mass parameter $c_{1}$. We see that in the axial sector the masses increase with $c_{1}$, which is the expected behavior for mesons. These results suggest the possibility of using the mass of the first axial-vector meson $a_{1}(1230)$ to fix the parameter $C_{0}$ for a given $\lambda$. Note, however, that the axialvector meson masses grow very fast and tend to diverge at a finite value of $c_{1}$. That value corresponds to the unphysical upper bound for the quark mass, described in the previous subsection.

\section{Spectrum of the pseudoscalar sector}

The pseudoscalar sector is special because it is described by a coupled system of differential equations (A14) and (A15). After performing the Fourier transform $\pi\left(x^{\mu}, z\right) \rightarrow$ $\pi\left(k^{\mu}, z\right)$ and $\varphi\left(x^{\mu}, z\right) \rightarrow \varphi\left(k^{\mu}, z\right)$ in both equations, where we have set $\square \rightarrow m_{\pi}^{2}$, we get the following equations:

$$
\begin{gathered}
e^{-A_{s}+\Phi} \partial_{z}\left(e^{A_{s}-\Phi} \partial_{z} \varphi\right)+g_{5}^{2} e^{2 A_{s}+2 \log v}(\pi-\varphi)=0, \\
-m_{\pi}^{2} \partial_{z} \varphi+g_{5}^{2} e^{2 A_{s}+2 \log v} \partial_{z} \pi=0 .
\end{gathered}
$$



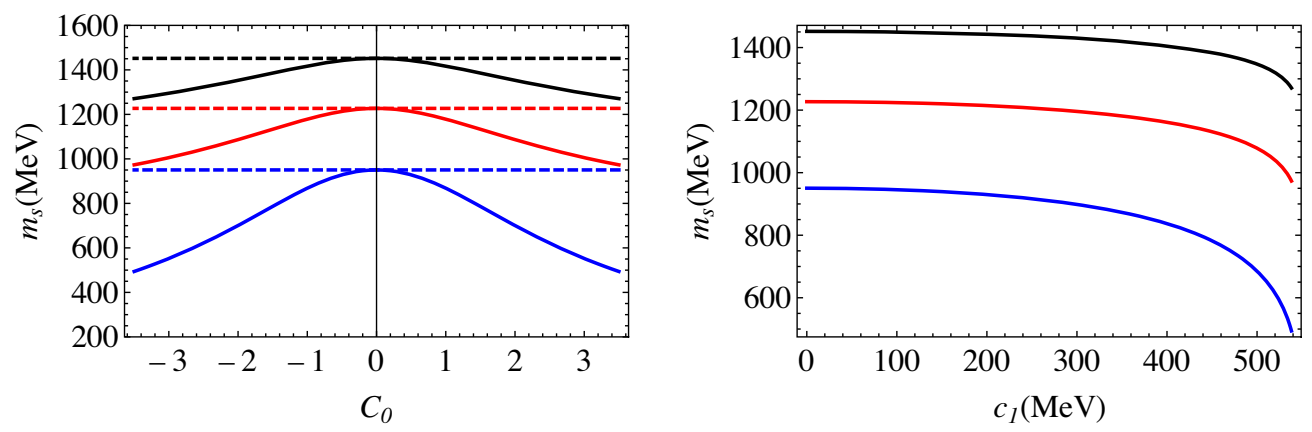

FIG. 6. Mass of the scalar mesons as a function of (left panel) $C_{0}$ and (right panel) $c_{1}$. Solid lines represent the results for $\lambda=-2$, dashed lines for $\lambda=0$. The results were obtained setting $\phi_{\infty}=(388 \mathrm{MeV})^{2}$.
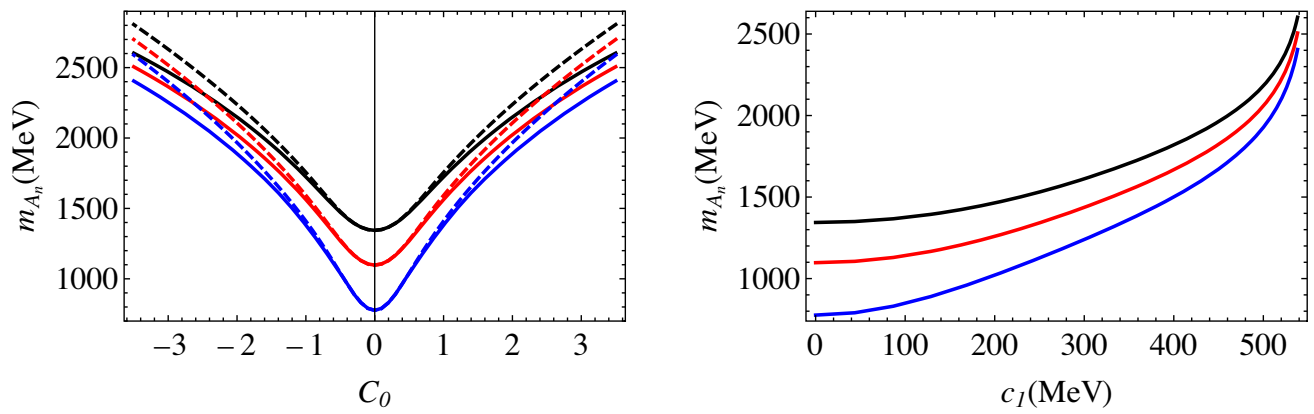

FIG. 7. Masses of axial-vector mesons as functions of (left panel) $C_{0}$ and (right panel) $c_{1}$. Solid lines represent the results for $\lambda=-2$, dashed lines for $\lambda=0$. The results were obtained setting $\phi_{\infty}=(388 \mathrm{MeV})^{2}$.

We follow [33] and decouple this system of equations. The decoupled equation is second order in the auxiliary field $\Pi=\partial_{z} \pi_{n}$ and takes the form

$$
\begin{aligned}
& -\partial_{z}^{2} \Pi+\partial_{z}\left(\Phi-A_{s}-\ln \beta\right) \partial_{z} \Pi \\
& \quad+\left(\partial_{z}^{2}\left(\Phi-A_{s}-\ln \beta\right)-m_{\pi}^{2}+\beta\right) \Pi=0,
\end{aligned}
$$

where we have introduced the function $\beta(z)=g_{5}^{2} e^{2 A_{s}} v^{2}$. Defining the function $2 B_{\pi}=A_{s}-\Phi+\log \beta$ and then introducing the transformation $\Pi=e^{-B_{\pi}} \psi_{\pi_{n}}$, we arrive at the Schrödinger equation

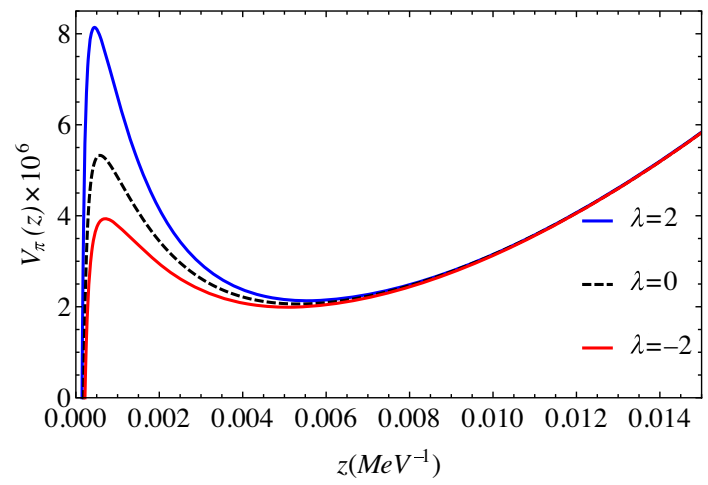

$$
-\partial_{z}^{2} \psi_{\pi_{n}}+V_{\pi} \psi_{\pi_{n}}=m_{\pi}^{2} \psi_{\pi_{n}},
$$

with the potential given by

$$
V_{\pi}=\left(\partial_{z} B_{\pi}\right)^{2}-\partial_{z}^{2} B_{\pi}+\beta .
$$

At this point it is interesting to display a plot of the potential (59). This is shown in the left panel of Fig. 8, where we observe potential wells emerging for different values of $\lambda$. These potential wells, however, are not deep enough to allow a light state in the spectrum. This is related to the fact that the soft wall backgrounds considered in this

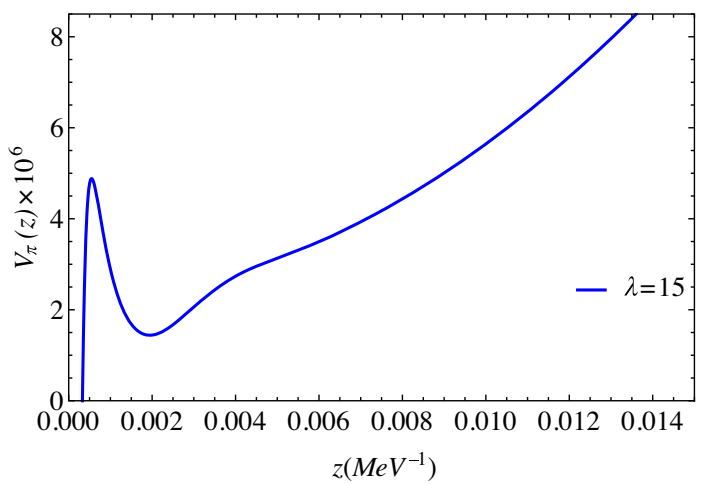

FIG. 8. (Left panel) Potential of the pseudoscalar Schrödinger equation in the NLSW model for $\lambda=2$ (blue line), $\lambda=0$ (dashed black line), and $\lambda=-2$ (red line). We have set $\phi_{\infty}=(388 \mathrm{MeV})^{2}$. (Right panel) The potential of the Gherghetta-Kapusta-Kelley (GKK) model. This figure was obtained using the same parameters as in [27]. 

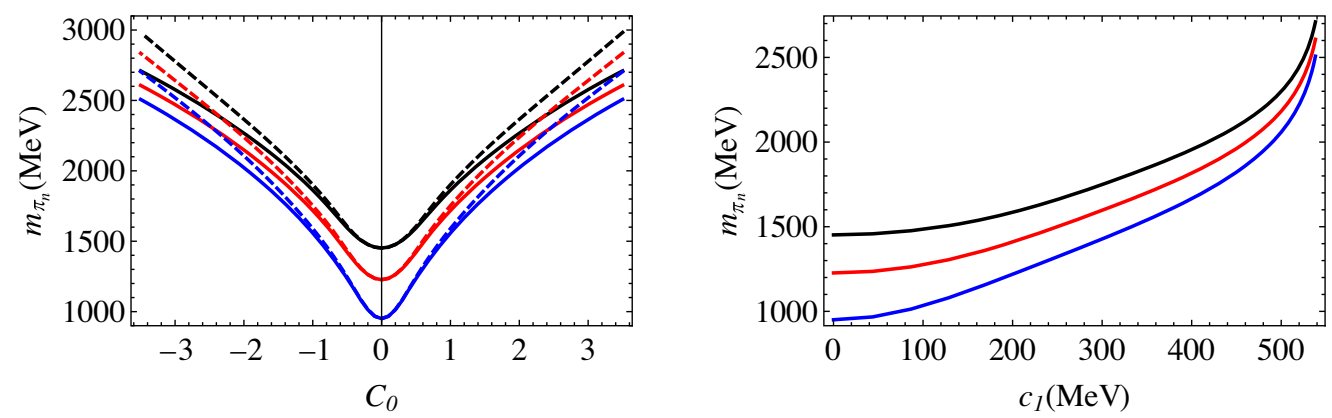

FIG. 9. Mass as a function of (left panel) $C_{0}$ and (right panel) $c_{1}$. Solid lines represent the results for $\lambda=-2$, dashed lines for $\lambda=0$. The results were obtained setting $\phi_{\infty}=(388 \mathrm{MeV})^{2}$.

work do not provide spontaneous symmetry breaking in the chiral limit, and therefore we would not expect pseudo-NG modes. In the right panel of Fig. 8 we display the potential obtained in the holographic model investigated in [11], where the potential well allows a light state in the spectrum, as described in [27].

In Fig. 9 we show the evolution of the pseudoscalar meson masses with the parameter $C_{0}$ (left panel) and $c_{1}$ (right panel) compared to the results of the linear soft wall model, which are plotted with dashed lines. Note that the masses increase with the quark mass parameter $c_{1}$, as expected in QCD. However, since there is an unphysical cutoff for the quark mass parameter $c_{1}$ the pseudoscalar meson masses grow very fast and tend to diverge when $c_{1}$ reaches the cutoff.

\section{E. Meson spectrum $(\lambda>0)$}

As described in Sec. III D 1, the spectrum of vector mesons is insensitive to the nonlinear potential, so it is identical to the linear case $(\lambda=0)$. Below we describe the spectrum of scalar, axial-vector, and pseudoscalar mesons for the case of positive $\lambda$, which corresponds to a Mexican hat potential in (18).

\section{Spectrum of the scalar sector}

The differential equation of the scalar sector, written in a Schrödinger form, was given in (51). The effect of going from negative to positive $\lambda$ is displayed in Fig. 5. We concluded that states become heavier for $\lambda>0$ compared to the cases $\lambda=0$ and $\lambda<0$. As described in the previous subsection, we consider the $f_{0}(980)$ state as the first scalar state and use that value to fix the parameter $C_{0}$ for a given $\lambda$. Our results for that parameter choice are displayed in Table II, labeled as NLSW, compared against the linear soft wall, the holographic model of [11], and experimental data. For $\lambda=7$ we obtain $C_{0}=0.3$ and find $c_{1}=142.4(\mathrm{MeV})$, which implies a large value for the quark mass. In Fig. 10 we show the evolution of the scalar meson masses as functions of the parameters $C_{0}$ (left panel) and $c_{1}$ (right panel). We observe that the masses increase with $C_{0}$ and $c_{1}$. The lower bound around $950 \mathrm{MeV}$ for the first scalar meson implies that we never reach the state $f_{0}(550)$, whose status is controversial anyway [29]. Meson mass increase with an increasing quark mass is a behavior expected in QCD, so we conclude that the model with $\lambda>0$ provides the best scenario. It is worth mentioning that the masses of scalar mesons do not vanish in the chiral limit.

\section{Spectrum of the axial-vector sector}

The Schrödinger equation for the axial-vector sector was given in Eq. (53), and this time we consider the case of $\lambda>0$. Solving that equation, we find the axial-vector meson masses. In Fig. 11 we show the evolution of the masses as functions of the parameters $C_{0}$ (left panel) and $c_{1}$ (right panel). The meson masses increase with the IR parameter $C_{0}$ and the $\mathrm{UV}$ quark mass parameter $c_{1}$. Meson mass increase with increasing quark mass is a behavior expected in QCD. In the chiral limit $c_{1} \rightarrow 0$ the masses of axial-vector mesons and vector mesons become degenerate.

In Table III we present our results for the parameter choice $\lambda=7, C_{0}=0.3$ fixed in the scalar sector. The results, labeled as NLSW, are compared with the linear soft wall model [7], the holographic model of [11], and experimental data [29]. We observe that our results are close to the results obtained in the linear soft wall model. This is explained by the smallness of the tachyon field for

TABLE II. The masses of the scalar mesons (in $\mathrm{MeV}$ ) obtained in the nonlinear soft wall model with $\lambda>0$ compared with the linear soft wall model [7], the holographic model of [11], and experimental data [29]. The values of the parameters are $\lambda=7$, $C_{0}=0.3$, and $\phi_{\infty}=(388 \mathrm{MeV})^{2}$.

\begin{tabular}{lrrcc}
\hline \hline$n$ & NLSW & SW [7] & GKK [11] & $f_{0}$ experimental [29] \\
\hline 1 & 980 & 950 & 799 & $980 \pm 10$ \\
2 & 1246 & 1227 & 1184 & $1350 \pm 150$ \\
3 & 1466 & 1452 & 1466 & $1505 \pm 6$ \\
4 & 1657 & 1646 & 1699 & $1724 \pm 7$ \\
5 & 1829 & 1820 & 1903 & $1992 \pm 16$ \\
6 & 1986 & 1978 & 2087 & $2103 \pm 8$ \\
7 & 2132 & 2125 & 2257 & $2314 \pm 25$ \\
8 & 2268 & 2262 & 2414 & \\
\hline \hline
\end{tabular}



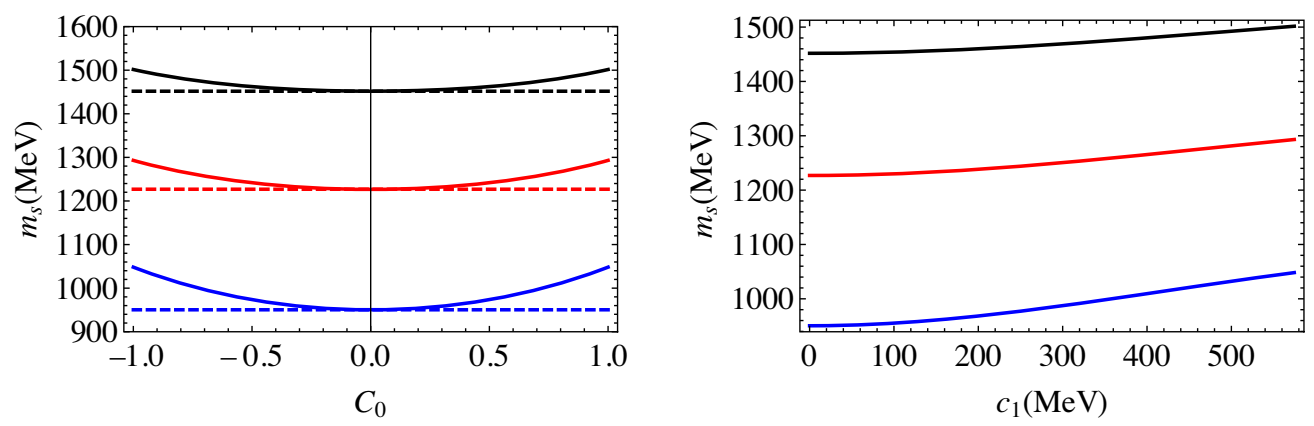

FIG. 10. Masses of the scalar mesons as a function of (left panel) $C_{0}$ and (right panel) $c_{1}$. Solid lines represent the result for $\lambda=2$, dashed lines for $\lambda=0$. The results were obtained by setting $\phi_{\infty}=(388 \mathrm{MeV})^{2}$.
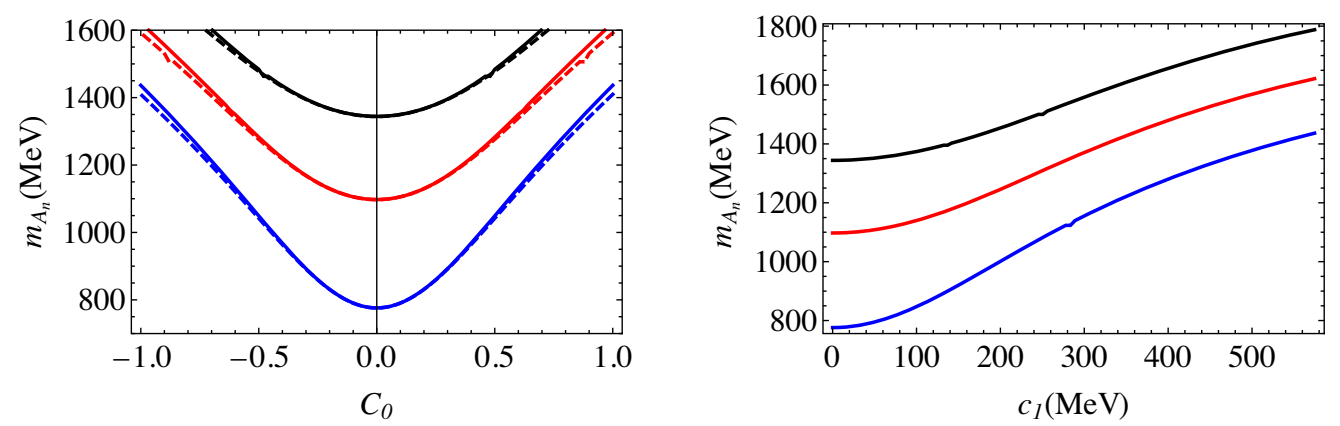

FIG. 11. Masses of the axial-vector mesons as a function of (left panel) $C_{0}$ and (right panel) $c_{1}$. Solid lines represent the result for $\lambda=2$, dashed lines for $\lambda=0$. The results were obtained setting $\phi_{\infty}=(388 \mathrm{MeV})^{2}$.

the parameters chosen to calculate the spectrum. For a small tachyon field the last term in the potential (54) is relatively small and the potential gets closer to the case of the linear soft wall model.

We point out that the spectrum of the vector and axialvector mesons presented in Tables I and III is not degenerate (the parameter $c_{1}$ is outside the chiral limit). In our model, the mass difference between the vector and axial-vector mesons at relatively large values of $c_{1}$ is purely a consequence of explicit chiral symmetry breaking, in contrast to QCD, where we expect another contribution coming from spontaneous chiral symmetry breaking.

TABLE III. The masses of the axial-vector mesons (in MeV) obtained in the nonlinear soft wall model compared to the linear soft wall model [7], the holographic model of [11], and experimental data [29]. The values of the parameters used are $\lambda=7, C_{0}=0.3$, and $\phi_{\infty}=(388 \mathrm{MeV})^{2}$.

\begin{tabular}{ccccc}
\hline \hline$n$ & NLSW & SW [7] & GKK [11] & $a_{1}$ experimental [29] \\
\hline 1 & 897 & 891 & 1185 & $1230 \pm 40$ \\
2 & 1172 & 1168 & 1591 & $1647 \pm 22$ \\
3 & 1398 & 1395 & 1900 & $1930_{-70}^{+30}$ \\
4 & 1594 & 1592 & 2101 & $2096 \pm 122$ \\
5 & 1770 & 1768 & 2279 & $2270_{-40}^{+55}$ \\
6 & 1930 & 1928 & & \\
7 & 2079 & 2077 & & \\
\hline \hline
\end{tabular}

\section{Spectrum of the pseudoscalar sector}

The pseudoscalar sector is described by the coupled equations (55) and (56). The system was decoupled and written in a Schrödinger form in Eq. (58), and this time we consider $\lambda>0$. Figure 12 shows our numerical results for the pseudoscalar meson masses as functions of the parameters $C_{0}$ (left panel) and $c_{1}$ (right panel). Again, we observe that the masses increase with the quark mass parameter $c_{1}$. Note that in the chiral limit $c_{1} \rightarrow 0$ the mass of the first pseudoscalar state has a finite value. This result can be interpreted as the absence of pseudo-NG bosons in the spectrum and supports the background analysis leading to a vanishing chiral condensate in the chiral limit.

As described previously, the potential well in the Schrödinger potential of the pseudoscalar sector, shown in the left panel of Fig. 8, is not deep enough to support the presence of a very light state. Therefore, the first state behaves as a pion resonance instead of a true pseudo-NG boson. This is in contrast to the Schrödinger potential of $[11,27]$, shown in the right panel of Fig. 8, which is deep enough to allow for a pseudo-NG boson. We will show in Sec. III F that all decay constants of the pseudoscalar mesons in our model go to zero in the chiral limit $c_{1} \rightarrow 0$, characterizing them as resonances.

What new feature does the holographic model in [11] have to allow for a pseudo-NG mode [27]? It is worth stressing that the main difference lies in the behavior of the 

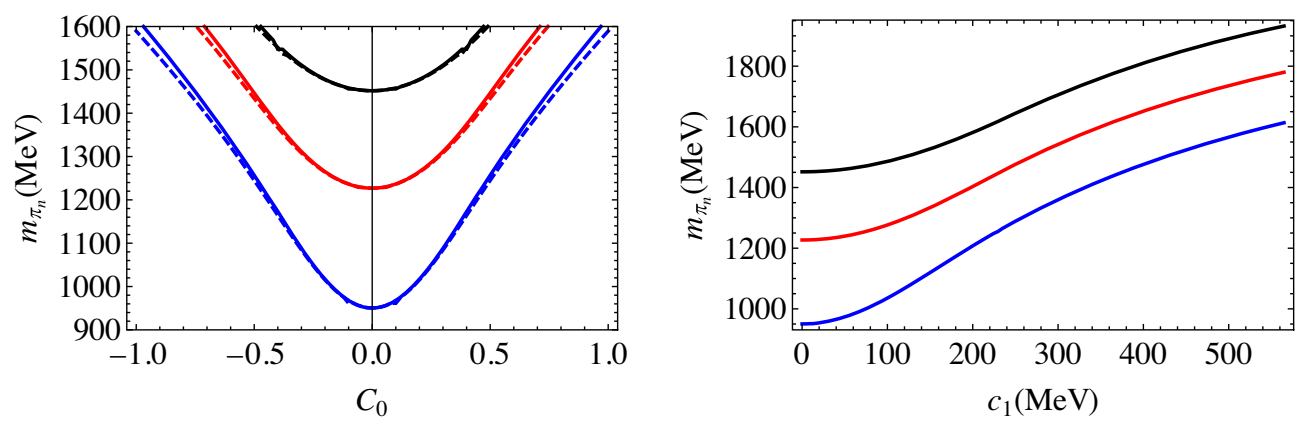

FIG. 12. Masses of the pseudoscalar mesons as a function of (left panel) $C_{0}$ and (right panel) $c_{1}$. Solid lines represent the result for $\lambda=2$, dashed lines for $\lambda=0$. The results were obtained by setting $\phi_{\infty}=(388 \mathrm{MeV})^{2}$.

dilaton field. In this model we consider a monotonically increasing positive dilaton, whereas the dilaton profile in [11] is negative in the UV and positive in the IR, with a minimum lying in the intermediate region; see Fig. 26 in Appendix B. This difference in the dilaton profile has an important consequence in the tachyon solution near the boundary. In the chiral limit the tachyon solution in our model vanishes, while the tachyon profile in $[11,27]$ is still nonzero. These two important differences have an effect on the Schrödinger potential that allows for a pseudo-NG mode in $[11,27]$. We remark, however, that we have followed a more conservative approach of taking a positive monotonically increasing dilaton to avoid instabilities in the meson spectrum. As described in Appendix B, the dilaton profile in [11] leads to instabilities in the scalar sector. It would be interesting if the model of [11] were to allow for an extension that avoids those instabilities.

We also note from Figs. 10 and 12 that in the chiral limit $c_{1} \rightarrow 0$ the masses of the pseudoscalar and scalar mesons reach the same values. We therefore conclude that the scalar and pseudoscalar mesons become degenerate in the chiral limit. As explained previously, the vector and axial-vector mesons also become degenerate in the chiral limit.

The spectrum obtained using the parameters $\lambda=7$ and $C_{0}=0.3$, fixed in the scalar sector, is displayed in Table IV. Our results, labeled as NLSW, are compared against the linear soft wall model [7], the holographic model of

TABLE IV. The masses of the pseudoscalar mesons (in MeV) obtained in the nonlinear soft wall model with $\lambda>0$ compared to the linear soft wall model [7], the holographic model of [27], and experimental data [29]. The values of the parameters are $\lambda=7$ and $C_{0}=0.3$.

\begin{tabular}{ccccc}
\hline \hline$n$ & NLSW & SW [7] & KBK [27] & $\pi$ experimental [29] \\
\hline 1 & $\ldots$ & $\ldots$ & 144 & 140 \\
2 & 1100 & 951 & 1557 & $1300 \pm 100$ \\
3 & 1321 & 1227 & 1887 & $1816 \pm 14$ \\
4 & 1518 & 1452 & 2090 & 2070 \\
5 & 1697 & 1646 & 2270 & 2360 \\
6 & 1861 & 1820 & 2434 & \\
7 & 2013 & 1980 & 2586 & \\
\hline \hline
\end{tabular}

$[11,27]$, and experimental data [29]. We also point out that the masses of scalar and pseudoscalar mesons shown in Tables II and IV are not degenerate (the parameter $c_{1}$ is outside the chiral limit).

We finish this subsection by showing in Fig. 13 the evolution of the parameters $C_{0}$ (left panel) and $c_{1}$ (right panel) when varying $\lambda$, matching the first scalar state with the meson $f_{0}(980)$. In the figure we observe why it is not possible to get small values for $c_{1}$ when $\lambda$ is small. To get a small quark mass, for example, $m_{q}=8 \mathrm{MeV}$, we would need $c_{1}=2.21 \mathrm{MeV}$, and therefore the value of $\lambda$ would be very large, i.e., $\lambda \approx 3 \times 10^{4}$, with the corresponding value for $C_{0} \approx 4.7 \times 10^{-3}$. Those results would have dramatic consequences in the spectrum because the IR parameter $C_{0}$ would be so small that the contribution to the Schrödinger equations would be negligible and the spectrum of scalar and pseudoscalar mesons, as well as the vector and axialvector mesons, would be degenerate.

\section{F. Decay constants}

In this subsection we calculate the decay constants of the vector, axial-vector, and pseudoscalar mesons. In holographic QCD models the meson decay constants are related to the normalization constants for the field perturbations; see, e.g., [34]. The normalization condition for the vector field is given by

$$
\int d z e^{A_{s}-\Phi} v_{m}(z) v_{n}(z)=\delta_{m n}
$$

where $A_{s}=-\ln z$ is the AdS warp factor, $\Phi(z)=\phi_{\infty} z^{2}$ is the dilaton, and $v_{n}$ is the vector meson mode related to the wave function by $v_{n}(z)=e^{-B_{V}} \psi_{v_{n}}(z)$. In turn, the wave function $\psi_{v_{n}}$ satisfies the Schrödinger equation (47). As described in Appendix A, the decay constants are related to $4 \mathrm{D}$ conserved currents and they are defined through the relations in Eq. (A21),

$$
F_{v_{n}}=-\left.\lim _{\epsilon \rightarrow 0} \frac{e^{A_{s}-\Phi}}{g_{5}} \partial_{z} v_{n}\right|_{z=\epsilon}=\frac{2}{g_{5}} N_{v_{n}}
$$



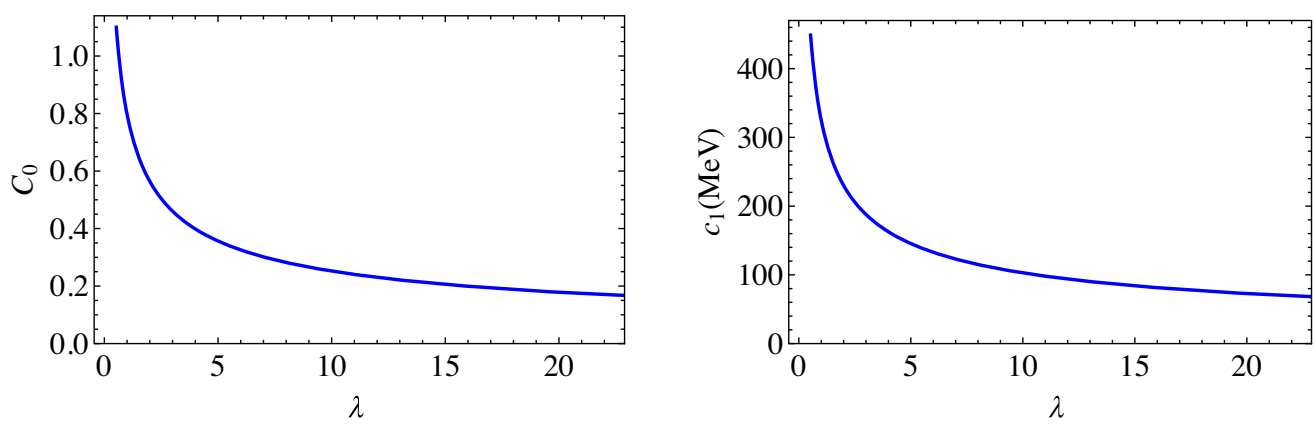

FIG. 13. Evolution of (left panel) $C_{0}$ as a function of $\lambda$ and (right panel) $c_{1}$ as a function of $\lambda$ when the first scalar state is matched to the meson $f_{0}(980)$. We remind the reader that $\phi_{\infty}=(388 \mathrm{MeV})^{2}$ fixed in the vector sector.
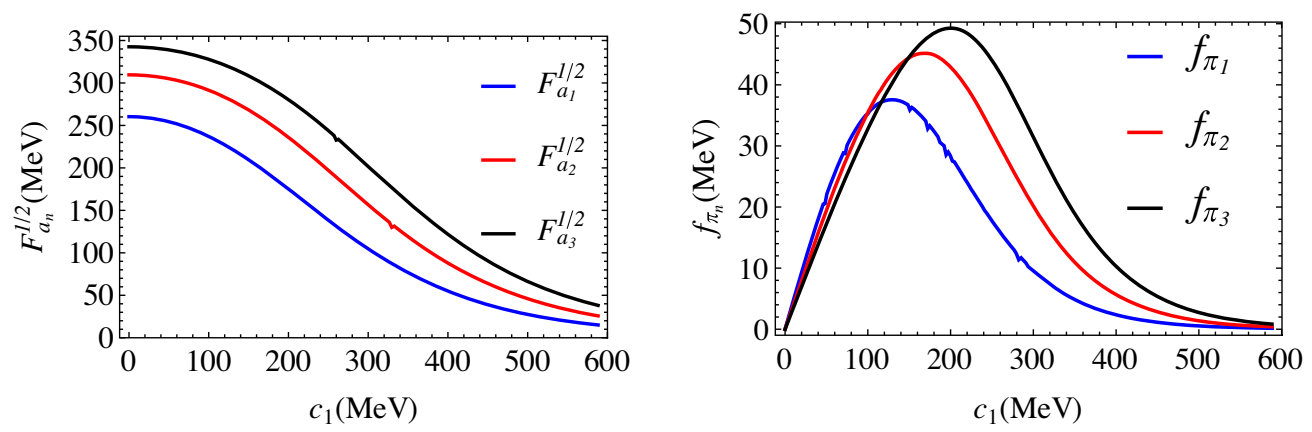

FIG. 14. Decay constants of the (left panel) axial-vector mesons and (right panel) pseudoscalar mesons as a function of $c_{1}$ obtained in the NLSW model for $\phi_{\infty}=(388 \mathrm{MeV})^{2}$ and $\lambda=2$.

The normalization constant $N_{v_{n}}$ appears as the UV coefficient near the boundary of a vector mode, i.e., $v_{n}(z)=$ $N_{v_{n}} z^{2}+\cdots$, satisfying the normalization condition (60). Thus, the decay constants are proportional to the normalization constants of the field perturbations. We follow the same procedure for the axial-vector sector, where the normalization condition is given by

$$
\int d z e^{A_{s}-\Phi} a_{m}(z) a_{n}(z)=\delta_{m n},
$$

where $a_{m}(z)=e^{-B_{A}} \psi_{a_{n}}(z)$ is the axial-vector model satisfying the UV behavior $a_{m}(z)=N_{a_{n}} z^{2}+\cdots$ and the normalization condition (62). $N_{a_{n}}$ is the normalization constant and $\psi_{a_{n}}$ is the solution of the Schrödinger equation (53). Thus, the decay constants are given by

$$
F_{a_{n}}=-\left.\lim _{\epsilon \rightarrow 0} \frac{e^{A_{s}-\Phi}}{g_{5}} \partial_{z} a_{n}\right|_{z=\epsilon}=\frac{2}{g_{5}} N_{a_{n}} .
$$

The problem of finding decay constants has been reduced to the problem of calculating normalization constants of the field perturbations (or, equivalently, wave functions). In the left panel of Fig. 14 we display the results for the decay constants of axial-vector mesons as functions of the quark mass parameter $c_{1}$. The decay constants do not vary significantly in the region of small $c_{1}$, but they decrease fast at large $c_{1}$ : approximately as $F_{a_{1}}^{1 / 2} \sim 1 / c_{1}^{b}$, with $b=2.6$, for the first state. In Table $\mathrm{V}$ we display our numerical results for the ground state $(n=1)$ decay constants for the parameter choices $\lambda=7$ and $C_{0}=0.3$.

For the pseudoscalar sector the normalization condition is given by [34]

$$
\int d z e^{A_{s}-\Phi} \beta(z)\left(\partial_{z} \pi_{m}\right)\left(\partial_{z} \pi_{n}\right)=m_{\pi_{n}}^{2} \delta_{m n}
$$

There are different approaches to calculate the decay constants of pseudoscalar mesons in the literature. First, we consider the prescription used in the hard wall model [4] (see also $[35,36])$. The authors considered the massless case, $m_{\pi}^{2}=0$. In that limit the pion decay constant can be extracted from the axial current correlator by the relation

TABLE V. The decay constants (in MeV) obtained in the nonlinear soft wall model compared to the result obtained in the linear soft wall model [7] and experimental results of PDG [29]. The results for $\lambda>0$ were obtained by setting $\lambda=7$ and $C_{0}=0.3$.

\begin{tabular}{lccc}
\hline \hline & NLSW $(\lambda>0)$ & SW [7] & Experimental [29] \\
\hline$F_{\rho}^{1 / 2}$ & 260.12 & 261 & $346.2 \pm 1.4$ \\
$F_{a_{1}}^{1 / 2}$ & 215.37 & 261 & $433 \pm 13$ \\
\hline \hline
\end{tabular}




$$
f_{\pi}^{2}=-\lim _{\epsilon \rightarrow 0} \frac{e^{A_{s}-\Phi}}{g_{5}^{2}} \partial_{z} A(0, \epsilon)
$$

where $A(0, \epsilon)$ is the non-normalizable solution for the axial-vector field dual to the 4D axial-vector current. We point out that the prescription is valid only in the case of $m_{\pi}^{2}=0$ and does not allow the investigation of pion resonances.

A prescription for calculating decay constants for the pion and their resonances was developed in [33]. Details on the derivation are given in Appendix A. The holographic dictionary for the decay constant may be written in the form

$$
f_{\pi_{n}}=-\left.\lim _{\epsilon \rightarrow 0} \frac{e^{A_{s}-\Phi}}{g_{5}} \partial_{z} \varphi_{n}(z)\right|_{z=\epsilon},
$$

where $\varphi_{n}(z)$ is the normalized wave function satisfying the normalization condition

$$
\int d z \frac{e^{A_{s}-\Phi}}{\beta(z)}\left(\partial_{z} \varphi_{m}\right)\left(\partial_{z} \varphi_{n}\right)=\delta_{m n}
$$

In terms of $\partial_{z} \pi_{n}$ the decay constant is obtained by plugging Eq. (56) into Eq. (66):

$$
f_{\pi_{n}}=-\left.\lim _{\epsilon \rightarrow 0} \frac{e^{A_{s}-\Phi}}{g_{5} m_{\pi_{n}}^{2}} \beta(z) \partial_{z} \pi_{n}(z)\right|_{z=\epsilon} .
$$

Hence, the normalization condition takes the form

$$
\int d z e^{A_{s}-\Phi} \beta(z)\left(\partial_{z} \pi_{m}\right)\left(\partial_{z} \pi_{n}\right)=m_{\pi_{n}}^{2} \delta_{m n}
$$

Finally, in terms of the Schrödinger wave function defined in Eq. (58), the decay constant takes the form

$$
f_{\pi_{n}}=-\left.\lim _{\epsilon \rightarrow 0} \frac{e^{\left(A_{s}-\Phi\right) / 2}}{g_{5} m_{\pi_{n}}^{2}} \beta^{1 / 2}(z) \psi_{\pi_{n}}(z)\right|_{z=\epsilon},
$$

where the normalization condition is given by

$$
\int d z \psi_{\pi_{m}}(z) \psi_{\pi_{n}}(z)=m_{\pi_{n}}^{2} \delta_{m n}
$$

In the end, the decay constant depends only on the normalization constant. Thus, the procedure above allows us to calculate decay constants of the fundamental state and its resonances. In our calculations for the pseudoscalar mesons we have used the three formulas described above to show the consistency of our numerical results. In the right panel of Fig. 14 we plot the decay constants as a function of the parameter $c_{1}$ of the first three pseudoscalar mesons. In the chiral limit the decay constants of all pseudoscalar states go to zero linearly, i.e., $f_{\pi_{n}} \sim c_{1}$, which agrees with the observed result for pion resonances in the hard wall model ([34]) and in QCD ([37]; see also [38]). On the other hand, as the quark mass increases the pseudoscalar meson decay constants display a nonmonotonic behavior and, in particular, go to zero in the heavy quark limit $c_{1} \rightarrow \infty$. It is worth mentioning that in the perturbative QCD approach for heavy quarks [39] one expects the behavior $f \sim 1 / \sqrt{M}$ for meson decay constants (see also [40]), where $M$ is the mass of the heavy mesons. In our case, the numerical data displayed in the right panel of Fig. 14 indicate the approximate behavior $f_{\pi_{1}} \sim 1 / c_{1}^{a}$, with $a=4.4$ for the first pseudoscalar state.

Finally, we note in the right panel of Fig. 14 a crossing of the different curves. The hierarchy between the decay constants changes when going from the regime of small quark mass to the regime of heavy quark mass. Numerical results are displayed in Table VI, for $\lambda=7$ and specific values of $C_{0}$. We see that the decay constants increase with the radial number for $C_{0}=0.4$, corresponding to $c_{1}=203.68 \mathrm{MeV}$, whereas for the case $C_{0}=0.1$, corresponding to $c_{1}=44.17 \mathrm{MeV}$, the decay constants decrease with the radial number. In the regime of small quark mass, the hierarchy $f_{\pi_{1}}>f_{\pi_{2}}>f_{\pi_{3}}$ was found in [33] for pion resonances in the hard wall model. We have found the same hierarchy in the (nonlinear) soft wall model in the regime of small quark mass and, interestingly, we have found an inversion of that hierarchy in the regime of heavy quark mass.

\section{NONLINEAR SOFT WALL MODELS WITH RUNNING MASS}

Recent works in holographic soft wall models have considered the possibility of a tachyon squared mass $m_{X}^{2}$ depending on the radial coordinate $z[41,42]$. The motivation for a tachyon running mass was to gain a nontrivial IR contribution to the tachyon differential equation and therefore find a richer dynamics. In holographic QCD a 5D running mass for the tachyon would correspond to the anomalous dimension for the 4D quark mass operator [22,42].

For the tachyon running mass we take the ansatz

$$
m_{X}^{2}(z)=-3-\phi_{c} z^{2}
$$

with $\phi_{C}>0$. The tachyon differential equation now becomes

$\left[z^{2} \partial_{z}^{2}-\left(3+2 \phi_{\infty} z^{2}\right) z \partial_{z}+3+\phi_{c} z^{2}\right] v-\frac{\lambda}{2} v^{3}=0$.

TABLE VI. The decay constants (in MeV) obtained in the nonlinear soft wall model. The results for $\lambda=7$ were obtained by setting $C_{0}=0.4\left(c_{1}=203.68 \mathrm{MeV}\right), C_{0}=0.1\left(c_{1}=44.17 \mathrm{MeV}\right)$, and $\phi_{\infty}=(388 \mathrm{MeV})^{2}$.

\begin{tabular}{lccc}
\hline \hline & $f_{\pi_{1}}$ & $f_{\pi_{2}}$ & $f_{\pi_{3}}$ \\
\hline NLSW $\left(C_{0}=0.4\right)$ & 27.20 & 40.84 & 47.36 \\
NLSW $\left(C_{0}=0.1\right)$ & 19.63 & 17.25 & 15.25 \\
\hline \hline
\end{tabular}




\section{A. Asymptotic analysis}

In the UV we consider again the Frobenius ansatz

$v(z)=c_{1} z+d_{3} z^{3} \ln z+c_{3} z^{3}+d_{5} z^{5} \ln z+c_{5} z^{5}+\ldots$

Plugging this ansatz into Eq. (73), we find the UV coefficients

$$
\begin{aligned}
d_{3}= & \frac{1}{4} c_{1}\left(c_{1}^{2} \lambda+4 \phi_{\infty}-2 \phi_{c}\right), \\
d_{5}= & \frac{1}{64} c_{1}\left(-c_{1}^{2} \lambda-4 \phi_{\infty}+2 \phi_{c}\right)\left(-c_{1}^{2} \lambda-12 \phi_{\infty}+2 \phi_{c}\right), \\
c_{5}= & \frac{1}{256}\left[-9 c_{1}^{5} \lambda^{2}-\left(-24 c_{1}^{3} \phi_{c}+56 c_{1}^{3} \phi_{\infty}+48 c_{1}^{2} c_{3}\right) \lambda\right. \\
& -12 c_{1} \phi_{c}^{2}+64 c_{1} \phi_{c} \phi_{\infty}-80 c_{1} \phi_{\infty}^{2} \\
& \left.-32 c_{3} \phi_{c}-192 c_{3} \phi_{\infty}\right] \ldots
\end{aligned}
$$

In the special case $c_{1}^{2} \lambda+4 \phi_{\infty}-2 \phi_{c}=0$ we have $d_{3}=$ $d_{5}=0$ and $c_{5}=\frac{1}{4} \phi_{c} c_{3}$, so we do not expect logarithmic terms, but there may be another solution besides the linear solution $v(z)=c_{1} z$ due to a nonzero $c_{3}$.

In the IR we work with the variable $y=1 / z$. Equation (73) becomes

$\left[\left(y \partial_{y}\right)^{2}+2\left(2+\phi_{\infty} y^{-2}\right)\left(y \partial_{y}\right)+\left(3+\phi_{c} y^{-2}\right)\right] v-\frac{\lambda}{2} v^{3}=0$.

We take again the power ansatz

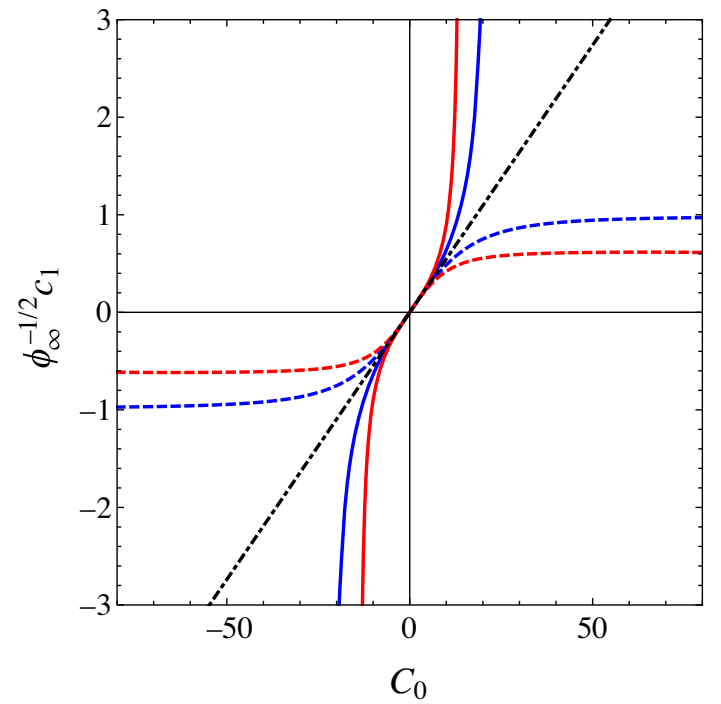

$$
v^{\mathrm{IR}}(y)=C_{0} y^{\alpha} .
$$

Plugging this ansatz into Eq. (76), we obtain the polynomial equation

$C_{0} y^{\alpha}\left(\alpha^{2}+4 \alpha+3\right)+C_{0} y^{\alpha-2}\left(2 \alpha \phi_{\infty}+\phi_{c}\right)-\frac{\lambda}{2} C_{0}^{3} y^{3 \alpha}=0$.

Again, we distinguish among three cases: $\alpha>-1, \alpha=-1$, and $\alpha<-1$. The last case is trivial because it leads to $C_{0}=0$.

In the case $\alpha>-1$ the second term dominates and we find

$$
\alpha=-\frac{\phi_{c}}{2 \phi_{\infty}}, \quad \phi_{c}<2 \phi_{\infty}
$$

This is a natural deformation of the regular solution found in the previous section. For $0>\alpha>-1$ the solution is actually divergent and admits the expansion

$v^{\mathrm{IR}}(y)=y^{\alpha}\left(C_{0}+C_{2} y^{\beta}+\ldots\right), \quad \beta=2-\frac{\phi_{c}}{\phi_{\infty}}$,

with

$$
C_{2}=-\frac{C_{0}^{3} \lambda}{4\left(\phi_{c}-2 \phi_{\infty}\right)}
$$

We will focus on the case $\phi_{c}=\phi_{\infty}$ where, in terms of $z$, the tachyon solution reads

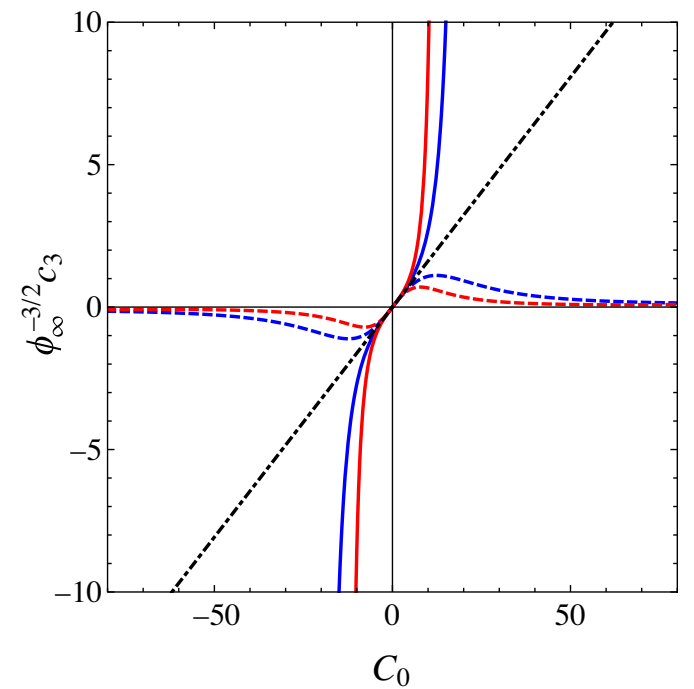

FIG. 15. Numerical results for (left panel) $c_{1}$ and (right panel) $c_{3}$ as functions of $C_{0}$ in units of $\sqrt{\phi_{\infty}}$. The blue and red solid lines (dashed lines) correspond to $\lambda=2$ and $\lambda=5(\lambda=-2$ and $\lambda=-5)$. The black dot-dashed line represents the case $\lambda=0$ (linear soft wall model with running mass). 


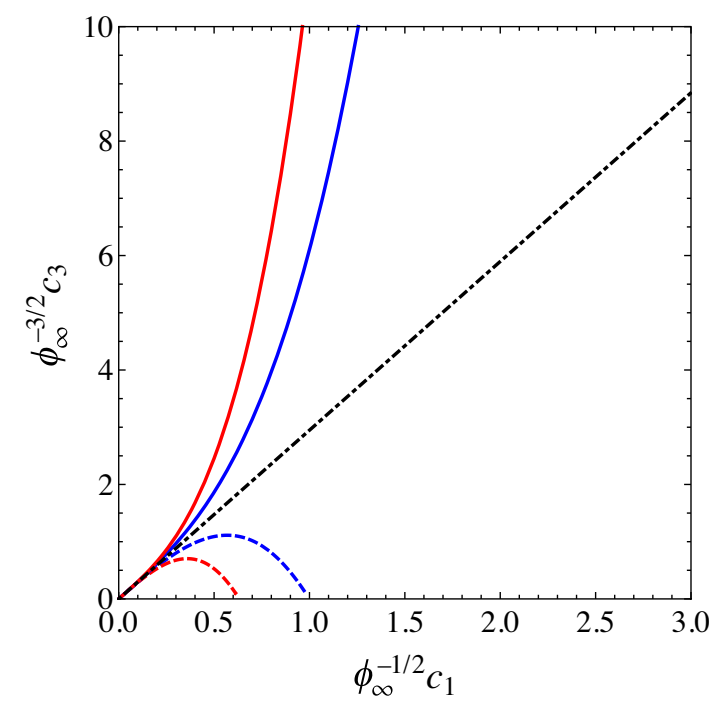

FIG. 16. VEV parameter $c_{3}$ as a function of the source parameter $c_{1}$, in units of $\sqrt{\phi_{\infty}}$, for different values of $\lambda$. The blue and red solid lines (dashed lines) correspond to $\lambda=2$ and $\lambda=5(\lambda=-2$ and $\lambda=-5)$. The black dot-dashed line represents the case $\lambda=0$ (linear soft wall model with running mass).

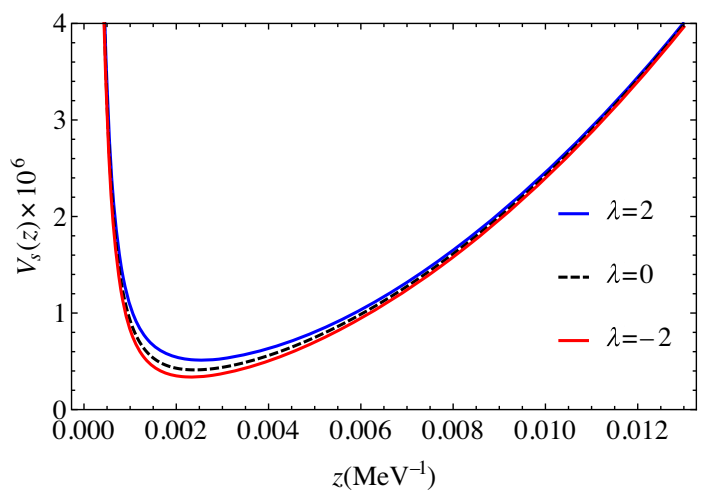

FIG. 17. Potential of the Schrödinger equation in the scalar sector for $\phi_{c}=\phi_{\infty}=(388 \mathrm{MeV})^{2}$ and three different values of the parameter $\lambda$.

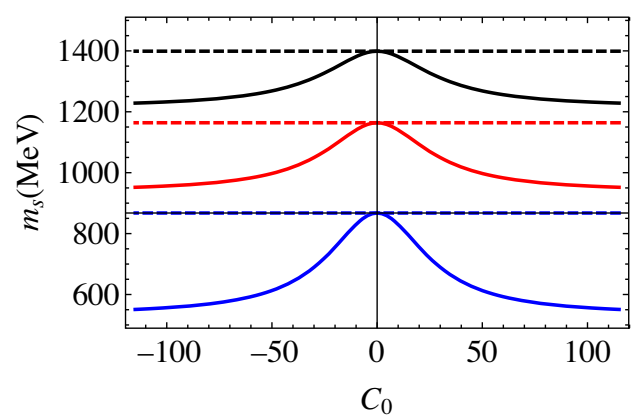

$v=C_{0} \sqrt{z}\left(1+\frac{C_{0}^{2} \lambda}{4 \phi_{\infty}} z^{-1}+\frac{3 C_{0}^{4} \lambda^{2}-10 \phi_{\infty}}{32 \phi_{\infty}^{2}} z^{-2}+\cdots\right)$.

This is a divergent solution depending on only one parameter, $C_{0}$. The IR leading behavior $v \propto \sqrt{z}$ was considered as an IR constraint in a previous approach [43].

In the special case $\alpha=-1$ the first term in Eq. (78) vanishes, whereas the second and third terms lead to the condition $-C_{0}^{2} \lambda+2 \phi_{c}-4 \phi_{\infty}=0$. This linear solution, i.e., $v(z)=C_{0} z$, is valid only for $\left(\lambda<0, \phi_{c}<2 \phi_{\infty}\right)$ or $\left(\lambda>0, \phi_{c}>2 \phi_{\infty}\right)$.

\section{Running mass with $\lambda=0$}

We finish this subsection by pointing out that the divergent solution (80) survives in the case $\lambda=0$. This corresponds to the linear soft wall model with running mass. In the case $\phi_{c}=\phi_{\infty}$ this solution has the following UV and IR behavior:

$$
\begin{gathered}
v^{\mathrm{UV}}(z)=c_{1} z+\frac{c_{1} \phi_{\infty}}{2} z^{3} \ln z+c_{3} z^{3}+\cdots, \\
v^{\mathrm{IR}}(z)=C_{0} \sqrt{z}\left(1-\frac{5}{16 \phi_{\infty}} z^{-2}+\cdots\right) .
\end{gathered}
$$

\section{B. Numerical solution}

The numerical results for the nonlinear soft wall model in the presence of a tachyon running mass are qualitatively similar to the case without the running mass. The main effect of the running mass will be extending the range for the IR parameter $C_{0}$. In particular, for the case $\lambda>0$ the upper bound $C_{0}^{2} \lambda<6$ found in the previous section is not present anymore.

We present numerical results for the case $\phi_{c}=\phi_{\infty}$ corresponding to the IR behavior (82). Figure 15 displays the UV parameters $c_{1}$ and $c_{3}$ as functions of the IR parameter $C_{0}$ for different values of $\lambda$. The case $\lambda=0$, represented by black dot-dashed lines, corresponds to the

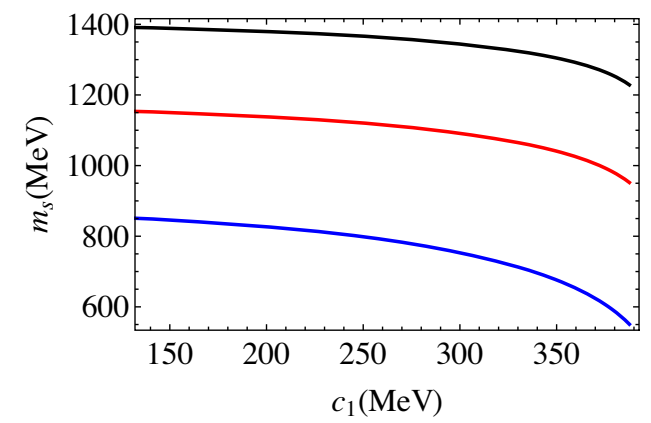

FIG. 18. Mass of the scalar mesons as a function of (left panel) $C_{0}$ and (right panel) $c_{1}$. Solid lines represent the result for $\lambda=-2$, dashed lines for $\lambda=0$. The results were obtained setting $\phi_{\infty}=\phi_{c}=(388 \mathrm{MeV})^{2}$. 

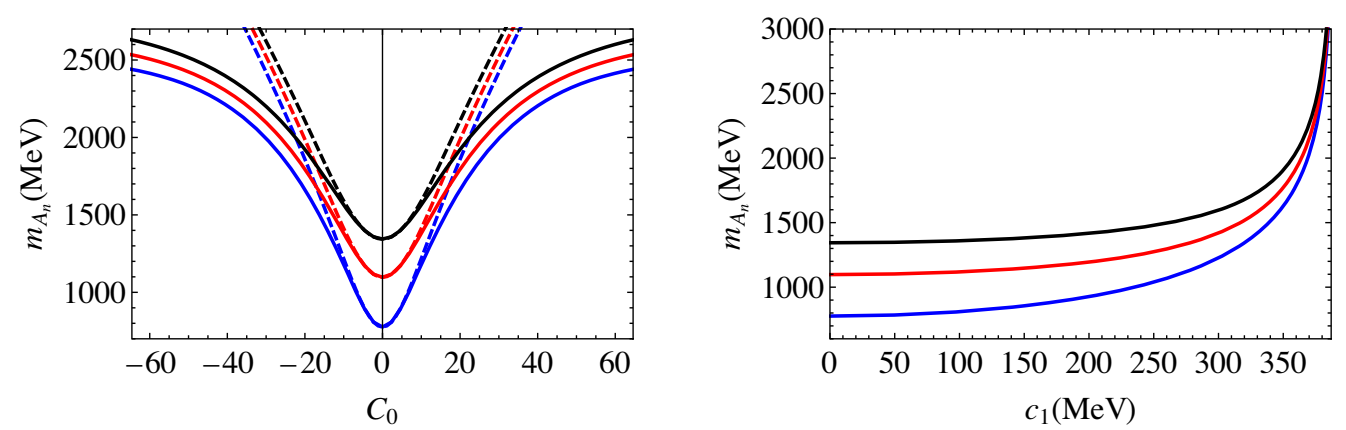

FIG. 19. Masses of axial-vector mesons as functions of (left panel) $C_{0}$ and (right panel) $c_{1}$. Solid lines represent the results for $\lambda=-2$, dashed lines for $\lambda=0$. The results were obtained setting $\phi_{\infty}=\phi_{c}=(388 \mathrm{MeV})^{2}$.
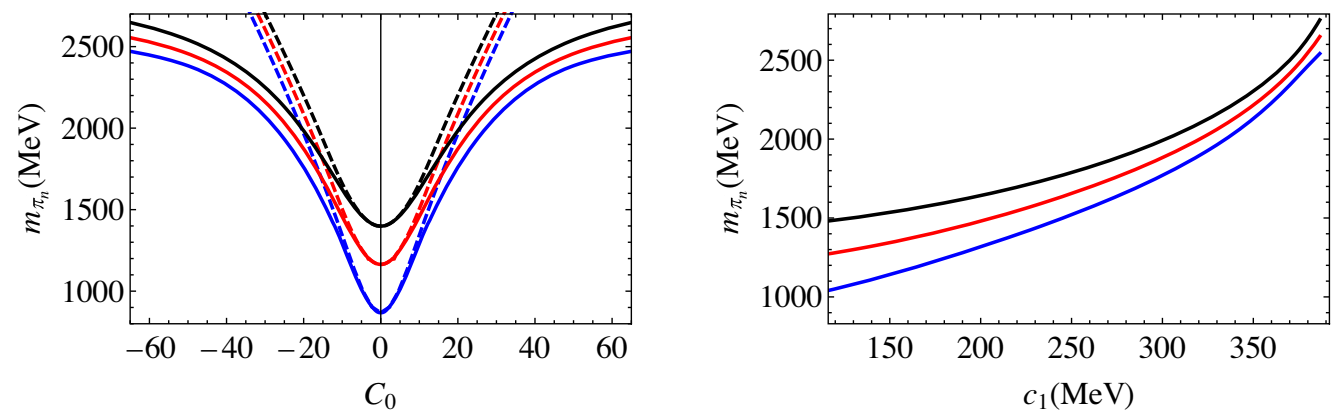

FIG. 20. Mass of the pseudoscalar mesons as a function of (left panel) $C_{0}$ and (right panel) $c_{1}$. Solid lines represent the result for $\lambda=-2$, dashed lines for $\lambda=0$. The results were obtained by setting $\phi_{\infty}=\phi_{c}=(388 \mathrm{MeV})^{2}$.
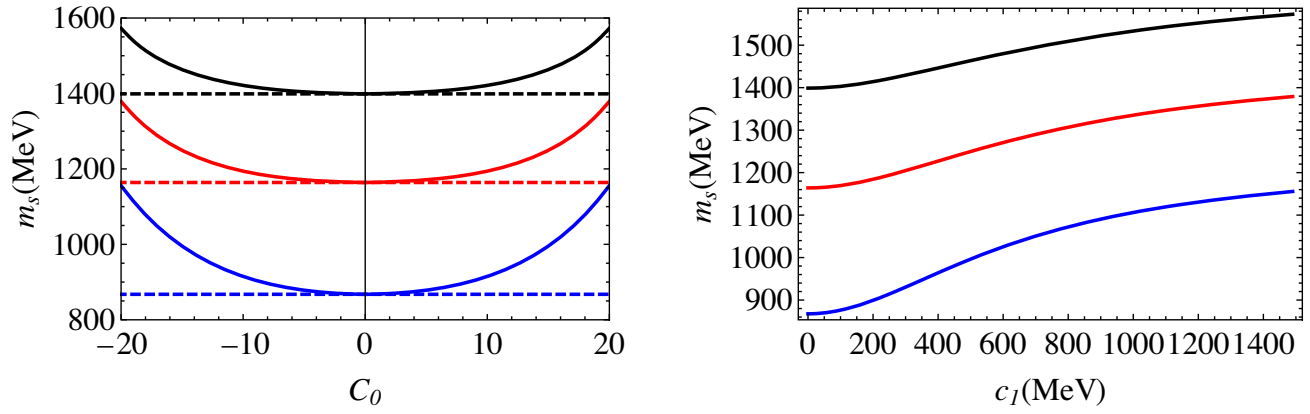

FIG. 21. Masses of scalar mesons as functions of (left panel) $C_{0}$ and (right panel) $c_{1}$. Solid lines represent the result for $\lambda=2$, dashed lines for $\lambda=0$. The results were obtained by setting $\phi_{\infty}=\phi_{c}=(388 \mathrm{MeV})^{2}$.

(linear) soft wall model with a running mass for the tachyon. Figure 16 displays $c_{3}$ as a function of $c_{1}$, which can be interpreted in terms of the $4 \mathrm{D}$ chiral condensate as a function of the quark mass, as described in Sec. III C. For the case $\lambda<0$ we note a decrease in the range of $c_{1}$ and $c_{3}$ compared to the case without running mass described in the previous section. For the case $\lambda>0$, despite having a bigger $C_{0}$ range, we do not notice a significant difference in $c_{3}$ vs $c_{1}$ compared to the case without the running mass. Again, we conclude that the case $\lambda>0$ provides the more realistic scenario for chiral symmetry breaking.

One of the motivations for considering a 5D running mass for the tachyon was to gain a nontrivial dynamics in the IR depending on the parameter $\phi_{c}$ in Eq. (72). However, we have found that the numerical results were very similar,
TABLE VII. Masses of the scalar mesons (in MeV) obtained in the nonlinear soft wall model with running mass compared to the results of Refs. [11,42] and the experimental results of PDG [29]. The values of the parameters used are $\lambda=7$ and $C_{0}=7.6$.

\begin{tabular}{ccccc}
\hline \hline$n$ & NLSW-RM & FLZ A [42] & GKK [11] & $f_{0}$ experimental [29] \\
\hline 1 & 980 & 586 & 799 & $980 \pm 10$ \\
2 & 1238 & 1346 & 1184 & $1350 \pm 150$ \\
3 & 1455 & & 1466 & $1505 \pm 6$ \\
4 & 1645 & 1743 & 1699 & $1724 \pm 7$ \\
5 & 1816 & 2232 & 1903 & $1992 \pm 16$ \\
6 & 1973 & 2420 & 2087 & $2103 \pm 8$ \\
7 & 2118 & & 2257 & $2314 \pm 25$ \\
8 & 2255 & & 2414 & \\
\hline \hline
\end{tabular}



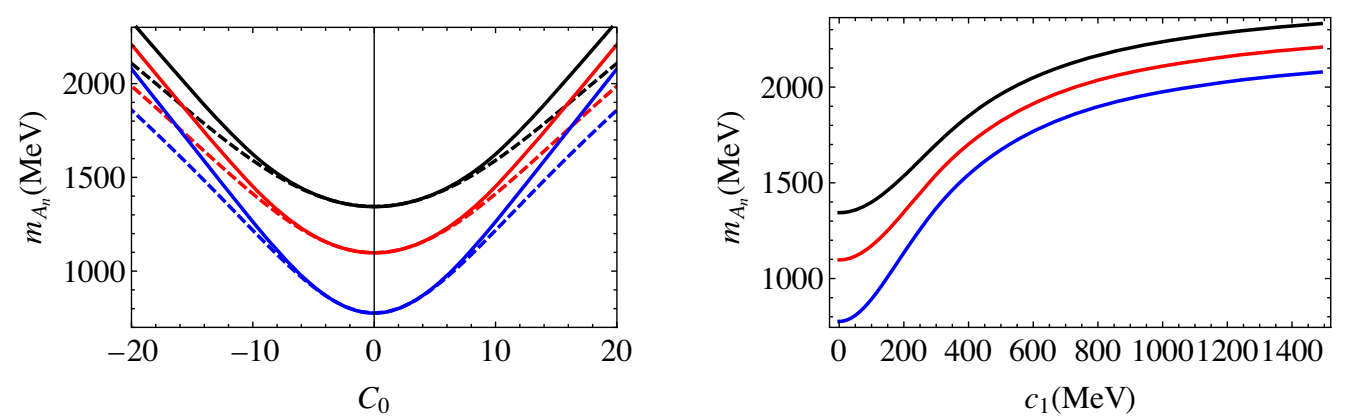

FIG. 22. Mass of the axial-vector mesons as a function of (left panel) $C_{0}$ and (right panel) $c_{1}$. Solid lines represent the results for $\lambda=2$, dashed lines for $\lambda=0$. The results were obtained setting $\phi_{\infty}=\phi_{c}=(388 \mathrm{MeV})^{2}$.

despite having this time a tachyon solution divergent in the IR. This may be related to the fact that the divergence is of the form $z^{\alpha}$ with $0<\alpha<1$. As in the case without running mass, in the chiral limit $c_{1} \rightarrow 0$ all the parameters go to zero. This is a negative result for nonlinear soft wall models because they do not describe spontaneous symmetry breaking in the chiral limit, in constrast to QCD.

\section{Meson spectrum $(\lambda<0)$}

As in the case without running mass, the vector meson is insensitive to the tachyon dynamics, and therefore we focus on the scalar, axial-vector, and pseudoscalar sectors.

\section{Spectrum of the scalar sector}

The equation of the scalar sector is again Eq. (50), but this time considering a running mass term $m_{X}^{2}(z)=$ $-3-\phi_{c} z^{2}$. We focus on the case $\phi_{\infty}=\phi_{c}$, where the tachyon solution diverges in the IR region as $v \sim \sqrt{z}$. We expect a different behavior of the potential in the Schrödinger equation, i.e., Eq. (51), and consequently a different spectrum. A plot of the potential is displayed in Fig. 17 for different values of $\lambda$; we observe the main difference between the models with negative, zero, or positive $\lambda$. In the case $\lambda<0$ it is possible to find a very

TABLE VIII. Masses of the axial-vector mesons (in $\mathrm{MeV}$ ) obtained in the nonlinear soft wall model with running mass compared to the results of GKK and Fang-Liang-Zhang (FLZ) of Refs. [11,42] and the experimental results [29]. The values of the parameters used are $\lambda=7, \quad C_{0}=7.6, \quad$ and $\phi_{\infty}=\phi_{c}=(388 \mathrm{MeV})^{2}$.

\begin{tabular}{ccccc}
\hline \hline$n$ & NLSW-RM & FLZ A [42] & GKK [11] & $a_{1}$ experimental [29] \\
\hline 1 & 1147 & 1121 & 1185 & $1230 \pm 40$ \\
2 & 1359 & 1608 & 1591 & $1647 \pm 22$ \\
3 & 1547 & 1922 & 1900 & $1930_{-70}^{+30}$ \\
4 & 1718 & 2156 & 2101 & $2096 \pm 122$ \\
5 & 1876 & 2352 & 2279 & $2270_{-40}^{+55}$ \\
6 & 2023 & 2526 & & \\
7 & 2161 & & & \\
\hline \hline
\end{tabular}

light state, just as in the model without running mass; see Sec. III D 2. However, as in that case, we follow a more conservative approach and consider $f_{0}(980)$ as the first scalar meson.

In Fig. 18 we show the mass of the scalar mesons as a function of the IR parameter $C_{0}$ (left panel). From this figure we observe that the scalar meson mass decreases with $C_{0}$ (solid lines), suggesting the possibility of a light state in the spectrum. The right panel of Fig. 18 shows the evolution of the scalar meson masses with the quark mass, i.e., $c_{1} \propto m_{q}$. All of the masses decrease as the quark masses increase, which is not expected in QCD. This pathology of the case $\lambda<0$ had arisen previously in the model without running mass and may be related to the absence of a minimum in the Higgs potential (18) when $\lambda<0$.

\section{Spectrum of the axial-vector sector}

The Schrödinger equation describing the axial-vector sector is the same as Eq. (53), this time with a running mass term $m_{X}^{2}(z)=-3-\phi_{c} z^{2}$. We display the results of the evolution of the mass (for the first three states) as a function of the parameter $C_{0}$ in the left panel of Fig. 19 with solid lines, while dashed lines represent the results for $\lambda=0$. From this figure we see that the mass increases as the parameter $C_{0}$ increases. The right panel of Fig. 19 displays the axial-vector meson masses as functions of the quark mass parameter $c_{1}$. The masses of the axial-vector mesons initially increase slowly with the quark masses but then all grow rapidly becoming divergent as the quark mass parameter reaches its upper bound. As described previously, an upper bound for the quark mass is not expected in QCD. In our model this upper bound arises as a saturation effect due to a negative quartic coupling $\lambda$ for the Higgs potential (18).

\section{Spectrum of the pseudoscalar sector}

The coupled equations of the pseudoscalar mesons are the same as Eqs. (55) and (56). Combining those equations, we reduced them into a Schrödinger form in Eq. (58). Using the same numerical procedure applied in the case without running mass, we are able to find the masses of pseudoscalar masses as functions of the parameters $C_{0}$ and 

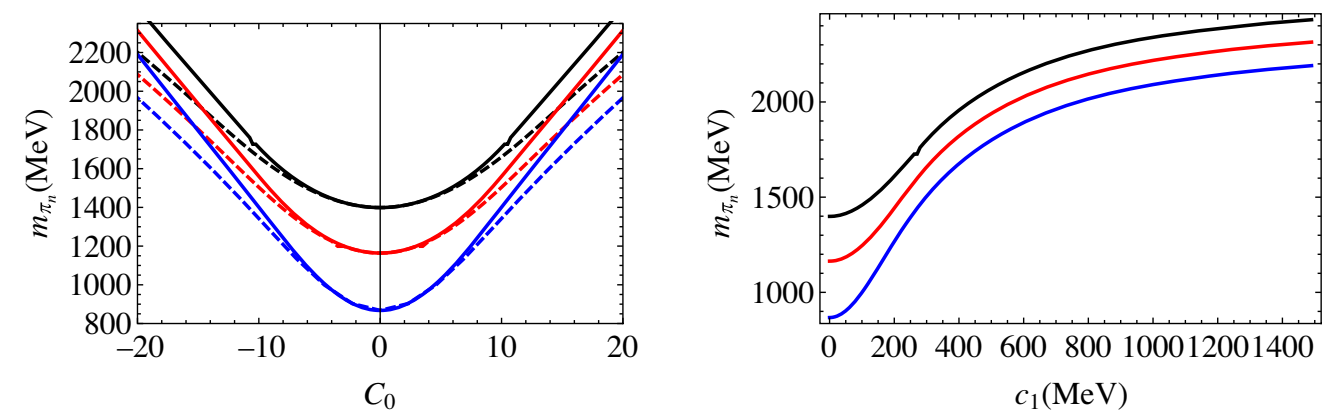

FIG. 23. Mass of the pseudoscalar mesons as a function of (left panel) $C_{0}$ and (right panel) $c_{1}$. Solid lines represent the result for $\lambda=2$, dashed lines for $\lambda=0$. The results were obtained by setting $\phi_{\infty}=\phi_{c}=(388 \mathrm{MeV})^{2}$.

$c_{1}$, We display the numerical results in Fig. 20. The left panel of the figure shows the variation of the masses as functions of $C_{0}$ with solid lines, while the results for $\lambda=0$ are represented with dashed lines; we see that the masses of pseudoscalar mesons always increase with $C_{0}$. We also point out that the mass increases faster close to $C_{0}=0$ and more slowly for large values of $C_{0}$. The right panel of Fig. 20 displays the evolution of the masses as a function of the quark mass parameter $c_{1}$. We observe that the masses of pseudoscalar mesons increase slowly in the region of small quark mass and faster in the intermediate and large quark mass region. Again, it seems that the masses diverge when the quark mass parameter $c_{1}$ reaches its upper bound.

\section{Meson spectrum $(\lambda>0)$}

\section{Spectrum of the scalar sector}

The potential of the Schrödinger equation in the scalar sector was displayed in Fig. 17 for $\lambda=2$. Our numerical results of the masses as functions of the parameter $C_{0}\left(c_{1}\right)$ are displayed in the left panel (right panel) of Fig. 21. We see that the scalar meson masses increase with both parameters. This allows us to use the same strategy implemented in Sec. III E 1 to fix the parameters; i.e., we use the state $f_{0}(980)$. Having fixed the parameters, we calculate the spectrum, which is displayed in Table VII as NLSW-RM. Although we are able to find a spectrum

TABLE IX. Masses of the pseudoscalar mesons (in MeV) obtained in the nonlinear soft wall model with running mass compared to the results of Kelley-Bartz-Kapusta (KBK) and FLZ of Refs. [27,42] and the experimental results [29]. The values of the parameters used are $\lambda=7, C_{0}=7.6$, and $\phi_{\infty}=\phi_{c}=(388 \mathrm{MeV})^{2}$.

\begin{tabular}{ccccc}
\hline$n$ & NLSW-RM & FLZ A [42] & KBK [27] & $\pi$ experimental [29] \\
\hline 1 & $\ldots$ & 139.6 & 144 & 140 \\
2 & 1301 & 1269 & 1557 & $1300 \pm 100$ \\
3 & 1479 & 1753 & 1887 & $1816 \pm 14$ \\
4 & 1642 & 2051 & 2090 & 2070 \\
5 & 1796 & 2277 & 2270 & 2360 \\
6 & 1942 & 2467 & 2434 & \\
7 & 2081 & & 2586 & \\
\hline \hline
\end{tabular}

compatible with experimental results, this does not guarantee a physical value for the quark mass. For example, the parameter choice $\lambda=7$ and $C_{0}=7.6$ corresponds to a very large value for the quark mass parameter $c_{1}$, even larger than the value obtained in the case without a running mass.

\section{Spectrum of the axial-vector sector}

The axial-vector sector is described by the same equations as were used in Sec. III D 3, this time with running mass term $m_{X}^{2}(z)=-3-\phi_{c} z^{2}$. The evolution of the masses as functions of the parameter $C_{0}\left(c_{1}\right)$ is displayed in the left panel (right panel) of Fig. 22. We see that the masses of axial-vector mesons increase slowly with $c_{1}$ and seem to reach asymptotic finite values in the limit $c_{1} \rightarrow \infty$. In Table VIII we display the results for the parameter choice $\left(\lambda=7, C_{0}=7.6\right)$, which were fixed previously in the scalar sector.

It is worth mentioning that the spectrum of axial-vector mesons is very different from the spectrum of the vector mesons (see Table I), which means that neither sector is degenerate. This nondegeneracy of the spectrum is enhanced by the tachyon running mass because the tachyon field becomes divergent in the IR. This is also a signal that chiral symmetry is never restored in the axial-vector excited states.

\section{Spectrum of the pseudoscalar sector}

The pseudoscalar mesons are described by the same equations as in Sec. III D 4, this time with running mass term $m_{X}^{2}(z)=-3-\phi_{c} z^{2}$. The evolution of the pseudoscalar meson masses as functions of the parameter $C_{0}\left(c_{1}\right)$ is displayed in the left panel (right panel) of Fig. 23. As in the axial-vector sector, the masses in the pseudoscalar sector increase slowly with $c_{1}$ and seem to reach asymptotic finite values in the limit $c_{1} \rightarrow \infty$. Again, in the chiral limit $c_{1} \rightarrow 0$ the mass of the lightest state does not vanish. This means that we do not have a pseudo-NG boson in the spectrum, and the pseudoscalar mesons behave simply as pion resonances.

Our numerical results for the parameter choice $\lambda=7$ and $C_{0}=7.6$ are displayed in Table IX as NLSW-RM. Since a 

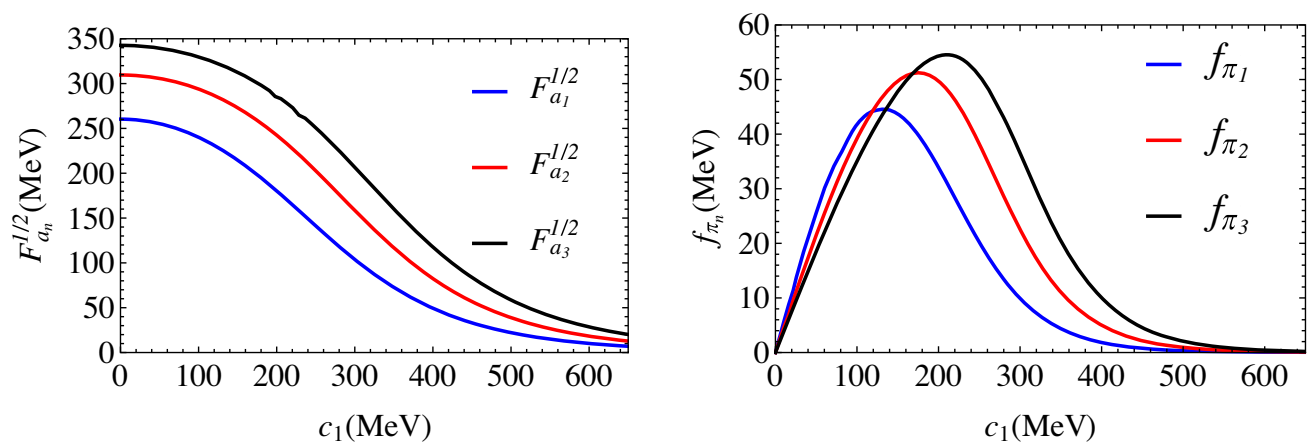

FIG. 24. Decay constants of the (left panel) axial-vector mesons and (right panel) pseudoscalar mesons as a function of $c_{1}$ obtained in the NLSW model with running masses for $\phi_{\infty}=\phi_{c}=(388 \mathrm{MeV})^{2}$ and $\lambda=2$.

TABLE X. The decay constants (in MeV) obtained in the nonlinear soft wall model with running mass compared to the results of Ref. [42] and the experimental results of PDG [29]. The results were obtained setting $\lambda=7, C_{0}=7.6$, and $\phi_{\infty}=\phi_{c}=(388 \mathrm{MeV})^{2}$.

\begin{tabular}{lccc}
\hline \hline & NLSW-RM $(\lambda>0)$ & FLZ A [42] & Experimental [29] \\
\hline$F_{\rho}^{1 / 2}$ & 260.12 & 296 & $346.2 \pm 1.4$ \\
$F_{a_{1}}^{1 / 2}$ & 152.78 & 389 & $433 \pm 13$ \\
\hline \hline
\end{tabular}

pseudo-NG boson is missing in the spectrum, the model at hand allows us to calculate only the spectrum of pion resonances. We also compare our results against the results available in Refs. [27,42] and experimental data [29].

\section{E. Decay constants}

In this subsection we calculate the decay constants of the vector, axial, and pseudoscalar mesons in the nonlinear soft wall model with running mass. As explained in Sec. III F, the decay constants are related to the normalization condition on the field perturbations. Again, we investigate the evolution of the decay constants of axial-vector and pseudoscalar mesons as a function of the quark mass parameter $c_{1}$. In the left panel of Fig. 24 we display the evolution of the decay constants of the first three axialvector mesons; the results are qualitatively similar to the case without running mass (see left panel of Fig. 14). In turn, the evolution of the decay constants of the first three

TABLE XI. Decay constants of the first three pseudoscalar mesons (in $\mathrm{MeV}$ ) obtained in the nonlinear soft wall model with running mass. The results were obtained by setting $\lambda=7$, $C_{0}=7.6\left(c_{1}=237.2 \mathrm{MeV}\right), C_{0}=0.2\left(c_{1}=4.25 \mathrm{MeV}\right)$, and $\phi_{\infty}=\phi_{c}=(388 \mathrm{MeV})^{2}$.

\begin{tabular}{lrrr}
\hline \hline & \multicolumn{1}{c}{$f_{\pi_{1}}$} & \multicolumn{1}{c}{$f_{\pi_{2}}$} & \multicolumn{1}{c}{$f_{\pi_{3}}$} \\
\hline NLSW-RM $\left(C_{0}=7.6\right)$ & 21.81 & 37.58 & 48.11 \\
NLSW-RM $\left(C_{0}=0.2\right)$ & 2.45 & 1.93 & 1.63 \\
\hline \hline
\end{tabular}

pseudoscalar mesons are displayed in the right panel of Fig. 24. Again, the results are qualitatively similar to the case without a running mass, which is displayed in the right panel of Fig. 14. In particular, we find again an inversion of hierarchy for the pseudoscalar meson decay constants. Considering the specific parameter choice $\lambda=7, C_{0}=7.6$ that fixes the mass of the first scalar state, we may calculate the decay constants of the first state of the vector and axialvector mesons. These results are displayed in Table $\mathrm{X}$ as NLSW-RM.

In Table XI we display the decay constants for the first three pseudoscalar mesons in the case $\lambda=7$ and specific values for $C_{0}$. These results show explicitly the inversion of hierarchy for the pseudoscalar decay constants when going from the regime of small quark mass to the regime of heavy quark mass; see the end of Sec. III F.

\section{DISCUSSION AND CONCLUSIONS}

In this work we investigated a nonlinear realization for chiral symmetry breaking in soft wall models based on a Higgs potential. Soft wall models allow for a more realistic description of the meson spectrum because a positive quadratic dilaton in the IR guarantees linear Regge trajectories. Solving the nonlinear differential equation of the tachyon, we found that the tachyon solution in the IR depends on only one parameter $C_{0}$, Integrating numerically the tachyon differential equation, we found nontrivial relations between the UV parameters $c_{1}$ and $c_{3}$ and the IR parameter $C_{0}$. Moreover, implementing the procedure of holographic renormalization, we obtained a dictionary for the 4D chiral condensate in terms of the VEV parameter $c_{3}$. This allowed us to find the evolution of the 4D chiral condensate with the quark mass. For the case $\lambda>0$, corresponding to a Mexican hat Higgs potential, we found that the chiral condensate grows nonlinearly with the quark mass, as expected in QCD. We found, however, that a nonlinear Higgs potential for the tachyon is not sufficient to provide spontaneous symmetry breaking in the chiral limit because the chiral condensate vanishes as the quark mass goes to zero. 
We calculated the spectrum of the scalar, vector, axialvector, and pseudoscalar mesons. The spectrum of vector mesons decoupled from the other sectors and their solutions was the same as in the linear soft wall model. We concluded that the case $\lambda>0$, corresponding to a Mexican hat Higgs potential, provides the most realistic scenario for the meson spectrum. The spectrum of the scalar mesons presented a pathology in the case $\lambda<0$ because the masses decreased with the quark mass. In the case $\lambda>0$ we found that it was possible to match the scalar state $f_{0}(980)$ state by fixing appropriately the parameters $\lambda$ and $C_{0}$. The analysis of the spectrum showed us that nonlinear soft wall models led to a nondegeneracy between vector and axial-vector mesons and also between scalar and pseudoscalar mesons. However, we found that it was not possible to fit the meson spectrum with a small quark mass parameter; see the end of Sec. III E 3. We calculated the decay constants of vector, axial-vector, and pseudoscalar mesons. The vector meson decay constants were insensitive to the tachyon dynamics and hence the quark mass. The axial-vector meson decay constants decreased with the quark mass, whereas the pseudoscalar meson decay constants presented a nonmonotonic behavior consistent with pion resonances. We found, in particular, that all of the pseudoscalar decay constants vanished in the chiral limit, indicating the absence of pseudo-NG bosons. A separate comment deserves mention concerning the decreasing behavior of the pseudoscalar decay constants in the regime of heavy quark mass, which agrees qualitatively with the perturbative QCD prediction; see the end of Sec. III F.

As an attempt to allow for spontaneous symmetry breaking in the chiral limit, we also investigated nonlinear soft wall models with a tachyonic running mass. The consequence of the running mass was to increase the interval of $C_{0}$ in relation to the model without a running mass. However, we realized that the results were qualitatively similar to the case without a running mass. Again, we found that $\lambda>0$ provides the most realistic scenario for the meson spectrum and decay constants, and we were able to again match the $f_{0}(980)$ state in the scalar sector by fixing appropriately the parameters. Again, we found that fixing the parameter to describe the meson spectrum led to a very large value for the quark mass. We found that the nondegeneracy between vector and axial-vector mesons was enhanced by the running mass, and the same was true for the scalar and pseudoscalar mesons.

Let us discuss briefly some other approaches to the problem of chiral symmetry in holographic QCD. The model proposed in [10] (see also [44]), which implemented the ideas of [21], claimed that the tachyon must blow up in the IR when the background has confinement properties. This statement may be related to the Coleman-Witten theorem [1]. As in our case, the IR tachyon solution in [10] depends on only one parameter. In contrast to our case, the model in [10] led to spontaneous chiral symmetry breaking in the chiral limit. Running mass models were considered in $[41,42,45]$ as an attempt to allow for spontaneous symmetry breaking in the chiral limit. We found, however, that in a consistent description of nonlinear soft wall models based on a Higgs potential, the tachyon running mass does not solve the lack of spontaneous symmetry breaking in the chiral limit. An interesting approach to the problem of chiral symmetry breaking is to consider a negative profile for the dilaton [9]. A negative dilaton profile has some issues; see, for instance, [46] for a discussion. For example, it would violate the null energy condition in the gravitational background [47]. Nevertheless, Chelabi et al. [9] proposed that the profile of the dilaton in the UV may be negative, while in the IR it must be positive in order to guarantee confinement and Regge-like behavior. They claim that in this way it is possible to describe spontaneous chiral symmetry breaking in the chiral limit.

In conclusion, nonlinear soft wall models based on a Higgs potential, with or without a tachyon running mass and a positive quadratic dilaton, do not provide spontaneous chiral symmetry breaking in the chiral limit. Consequently, there are no pseudo-NG bosons in the spectrum of pseudoscalar mesons. This conclusion is supported by the study of masses and decay constants in the region of small quark mass. We found, however, in the case of $\lambda>0$ a very reasonable behavior for the chiral condensate in the regime of large quark mass, similar to the behavior expected in QCD. For the case $\lambda>0$ we also found a reasonable behavior for all meson masses as growing functions of the quark mass. Interestingly, we found that the decay constants of axial-vector mesons and pseudoscalar mesons decreased in the regime of heavy quark mass. This behavior is also expected in QCD and encourages us to continue the investigation of nonlinear soft wall models. Finally, a natural extension of this work would be the investigation of backreacted Einstein-dilaton backgrounds, where the confinement criterion is satisfied. This would allow for a consistent description of confinement and chiral symmetry breaking in a minimal holographic setup.

\section{ACKNOWLEDGMENTS}

The authors would like to thank Carlisson Miller for the stimulating discussions during the early stages of this project. The authors would also like to acknowledge the very useful conversations with Saulo Diles, Diego Rodrigues, Jonathan Shock, and Dimitrios Zoakos during the development of this work. A. B.-B. would like to thank the organizers of WONPAQCD 2019 for providing a stimulating environment. The work of A. B.-B. is partially funded by Conselho Nacional de Desenvolvimento Científico e Tecnológico (CNPq) Grants No. 306528/2018-5 and No. 434523/2018-6. L. A. H. M. has financial support from Coordenação de Aperfeiçoamento do Pessoal de Nível Superior-Programa Nacional de Pós-Doutorado [(PNPD/ CAPES) Brazil]. 


\section{APPENDIX A: EQUATIONS OF MOTION AND DECAY CONSTANTS}

In this Appendix we write details about the derivation of the equations of motion used to calculate the meson spectrum as well as the holographic dictionary for meson decay constants. We will expand the action (19) up to second order on the fields and take the $5 \mathrm{D}$ metric as in Eq. (1).

We follow [34] (see also [48]). For simplicity, we take $N_{f}=2$ and assume isospin symmetry $\left(m_{u}=m_{d}\right)$. First, we decompose the bifundamental field $X$ in the form

$$
X=e^{2 i \pi^{a} T^{a}}\left(\frac{1}{2} v(z)+S\right)
$$

where $\pi^{a}\left(x^{\mu}, z\right)$ is the pseudoscalar field, $T^{a}$ are the generators of $S U(2)$, and $S\left(x^{\mu}, z\right)$ is the scalar fluctuation related to the scalar mesons. We also rewrite the fluctuation for the gauge fields as

$$
A_{m}^{(L / R)}=V_{m} \pm A_{m}
$$

where $V^{m}=V_{a}^{m} T^{a}$ and $A^{m}=A_{a}^{m} T^{a}$ are the vector and axial fields, respectively. Plugging Eqs. (A1) and (A2) into the action (19) and expanding on the fields $\pi^{a}, V_{a}^{m}$, and $A_{a}^{m}$ up to second order, we obtain $S=S_{0}+S_{2}+\cdots$, with $S_{0}$ the effective 1D action for the background $v(z)$ and

$$
\begin{aligned}
S_{2}= & -\int d x^{5} \sqrt{-g} e^{-\Phi}\left[2\left(\partial_{m} S\right)^{2}+2 m_{X}^{2} S^{2}+3 \lambda v^{2}(z) S^{2}\right. \\
& +\frac{1}{4 g_{5}^{2}} v_{a}^{m n} v_{m n}^{a}+\frac{1}{4 g_{5}^{2}} a_{a}^{m n} a_{m n}^{a} \\
& \left.+\frac{1}{2} v^{2}(z)\left(\partial_{m} \pi_{a}-A_{m, a}\right)^{2}\right]
\end{aligned}
$$

the action describing the kinetic terms for the 5D field fluctuations $\pi^{a}, V_{a}^{m}$, and $A_{a}^{m}$. We have defined the Abelian tensors

$v_{m n}^{a}=\partial_{m} V_{n}^{a}-\partial_{n} V_{m}^{a}, \quad a_{m n}^{a}=\partial_{m} A_{n}^{a}-\partial_{n} A_{m}^{a}$.

To obtain the Euler-Lagrange equations, we write the action as $S_{2}=\int d x^{5} \mathcal{L}_{2}$. The variation takes the form

$$
\begin{aligned}
\delta S_{2}= & \int d^{5} x\left[\left(\frac{\partial \mathcal{L}_{2}}{\partial S}-\partial_{m} P_{S}^{m}\right) \delta S+\left(\frac{\partial \mathcal{L}_{2}}{\partial V_{l}^{a}}-\partial_{m} P_{V, a}^{m l}\right) \delta V_{l}^{a}\right. \\
& \left.+\left(\frac{\partial \mathcal{L}_{2}}{\partial A_{l}^{a}}-\partial_{m} P_{A, a}^{m l}\right) \delta A_{l}^{a}+\left(\frac{\partial \mathcal{L}_{2}}{\partial \pi^{a}}-\partial_{m} P_{\pi, a}^{m}\right) \delta \pi^{a}\right] \\
& +\int d x^{5} \partial_{m}\left(P_{S}^{m} \delta S+P_{V, a}^{m l} \delta V_{l}^{a}+P_{A, a}^{m l} \delta A_{l}^{a}+P_{\pi, a}^{m} \delta \pi^{a}\right),
\end{aligned}
$$

where $P_{S}^{m}, P_{V, a}^{m l}, P_{A, a}^{m l}$, and $P_{\pi, a}^{m}$ are the conjugate momenta associated with the scalar, vector, axial-vector, and pseudoscalar fields, respectively. Explicitly, the conjugate momenta are given by

$$
\begin{aligned}
P_{S}^{m} & =\frac{\partial \mathcal{L}_{2}}{\partial\left(\partial_{m} S\right)}=-4 e^{-\phi} \sqrt{-g} \partial^{m} S, \\
P_{V, a}^{m l} & =\frac{\partial \mathcal{L}_{2}}{\partial\left(\partial_{m} V_{l}^{a}\right)}=-\frac{e^{-\phi}}{g_{5}^{2}} \sqrt{-g} v_{a}^{m l}, \\
P_{A, a}^{m l} & =\frac{\partial \mathcal{L}_{2}}{\partial\left(\partial_{m} A_{l}^{a}\right)}=-\frac{e^{-\phi}}{g_{5}^{2}} \sqrt{-g} a_{a}^{m l}, \\
P_{\pi, a}^{m} & =\frac{\partial \mathcal{L}_{2}}{\partial\left(\partial_{m} \pi^{a}\right)}=-e^{-\phi} \sqrt{-g} v^{2}(z)\left(\partial^{m} \pi^{a}-A^{m, a}\right) .
\end{aligned}
$$

In turn, the derivatives of the Lagrangian are

$$
\begin{aligned}
& \frac{\partial \mathcal{L}_{2}}{\partial S}=-e^{-\phi} \sqrt{-g}\left[4 m_{X}^{2}-6 v^{2}(z)\right] S, \quad \frac{\partial \mathcal{L}_{2}}{\partial V_{l}^{a}}=-0, \\
& \frac{\partial \mathcal{L}_{2}}{\partial A_{l}^{a}}=e^{-\phi} \sqrt{-g} v^{2}(z)\left(\partial^{l} \pi^{a}-A^{l, a}\right), \quad \frac{\partial \mathcal{L}_{2}}{\partial \pi^{a}}=0 .
\end{aligned}
$$

From these results we find that the equation of motion of the vector sector takes the form

$$
\frac{e^{\phi}}{\sqrt{-g}} \partial_{m}\left(e^{-\phi} \sqrt{-g} v_{a}^{m l}\right)=0
$$

The Abelian field strength was defined in Eq. (A4). Working in the axial gauge $V_{z}^{a}=0$, we get

$$
e^{-A_{s}+\Phi} \partial_{z}\left(e^{A_{s}-\Phi} \partial_{z} V_{\nu, a}\right)+\square V_{\nu, a}=0 .
$$

The equation of motion of the scalar sector takes the form

$$
\frac{e^{\phi}}{\sqrt{-g}} \partial_{m}\left(e^{-\phi} \sqrt{-g} g^{m n} \partial_{n} S\right)-\left(m_{X}^{2}+\frac{3}{2} \lambda v^{2}\right) S=0,
$$

which may be written in the form

$$
e^{-3 A_{s}+\Phi} \partial_{z}\left(e^{3 A_{s}-\Phi} \partial_{z} S\right)+\square S-e^{2 A_{s}}\left(m_{X}^{2}+\frac{3}{2} \lambda v^{2}\right) S=0
$$

The remaining equations of motion are

$$
\begin{aligned}
\frac{e^{\phi}}{\sqrt{-g}} \partial_{m}\left(e^{-\phi} \sqrt{-g} a_{a}^{m l}\right)+v^{2}(z) g_{5}^{2}\left(\partial^{l} \pi^{a}-A^{l, a}\right) & =0, \\
\partial_{m}\left[e^{-\phi} \sqrt{-g} v^{2}(z) g_{5}^{2}\left(\partial^{m} \pi^{a}-A^{m, a}\right)\right] & =0 .
\end{aligned}
$$


We work in the axial gauge, $A_{z}^{a}=0$, and decompose the gauge field as $A_{b}^{\mu}=A_{b \perp}^{\mu}+\partial^{\mu} \varphi_{b}$, where $A_{b \perp}^{l}$ is the transverse part and $\partial^{\mu} \varphi_{b}$ the longitudinal part. The tranverse part leads to the equation of motion for the axial-vector sector

$e^{A_{s}-\Phi} \partial_{z}\left(e^{A_{s}-\Phi} \partial_{z} A_{\perp}^{\mu, a}\right)+\square A_{\perp}^{\mu, a}-v^{2}(z) g_{5}^{2} e^{2 A_{s}} A_{\perp}^{\mu, a}=0$,

For the pseudoscalar sector we find the coupled equations

$$
\begin{gathered}
e^{A_{s}-\Phi} \partial_{z}\left(e^{A_{s}-\Phi} \partial_{z} \varphi^{a}\right)+v^{2}(z) g_{5}^{2} e^{2 A_{s}}\left(\pi^{a}-\varphi^{a}\right)=0, \\
-\partial_{z} \square \varphi^{a}+v^{2}(z) g_{5}^{2} e^{2 A_{s}} \partial_{z} \pi^{a}=0 .
\end{gathered}
$$

The dictionary for the decay constants is obtained from the holographic currents. The latter arise in the surface term of Eq. (A5), which may be written as

$$
\begin{aligned}
\delta S_{2}^{\mathrm{B} d y}= & -\int d x^{4}\left(\left\langle J_{s}\right\rangle(\delta S)+\left\langle J_{V, a}^{\mu}\right\rangle\left(\delta V_{\mu}^{a}\right)\right. \\
& \left.+\left\langle J_{A, a}^{\mu}\right\rangle\left(\delta A_{\mu}^{a}\right)+\left\langle J_{\pi, a}\right\rangle\left(\delta \pi^{a}\right)\right)_{z=\epsilon} .
\end{aligned}
$$

The VEV of $4 \mathrm{D}$ operators appearing in Eq. (A16) is defined by

$$
\begin{aligned}
\left\langle J_{s}\right\rangle & =P_{s}^{z}=-4 e^{-\phi} \sqrt{-g} \partial^{z} S, \\
\left\langle J_{V, a}^{\mu}\right\rangle & =P_{V, a}^{z \mu}=-\frac{e^{-\Phi}}{g_{5}^{2}} \sqrt{-g} v^{z \mu}, \\
\left\langle J_{A, a}^{\mu}\right\rangle & =P_{A, a}^{z \mu}=-\frac{e^{-\Phi}}{g_{5}^{2}} \sqrt{-g} a^{z \mu}, \\
\left\langle J_{\pi, a}\right\rangle & =P_{\pi, a}^{z}=-e^{-\Phi} \sqrt{-g} v^{2}(z)\left(\partial \pi^{a}-A^{z, a}\right) .
\end{aligned}
$$

We identify $\left\langle J_{V, a}^{\mu}\right\rangle$ and $\left\langle J_{A, a}^{\mu}\right\rangle$ as the holographic vector and axial currents leading to the meson decay constants. The next step is to decompose the fields into their KaluzaKlein modes as follows:

$$
\begin{aligned}
S(x, z) & =\sum_{n=0}^{\infty} s_{n}(z) \hat{S}_{n}(x), \\
V_{a}^{\mu}(x, z) & =g_{5} \sum_{n=0}^{\infty} v_{a, n}(z) \hat{V}_{a, n}^{\mu}(x), \\
A_{a}^{\mu, \perp}(x, z) & =g_{5} \sum_{n=0}^{\infty} a_{a, n}(z) \hat{A}_{a, n}^{\mu}(x), \\
\pi_{a}(x, z) & =g_{5} \sum_{n=0}^{\infty} \pi_{a, n}(z) \hat{\pi}_{a, n}(x), \\
\varphi_{a}(x, z) & =g_{5} \sum_{n=0}^{\infty} \varphi_{a, n}(z) \hat{\pi}_{a, n}(x) .
\end{aligned}
$$

Plugging Eq. (A18) into the vector and axial-vector currents (A17), we obtain the expansions

$$
\begin{aligned}
\left\langle J_{V, a}^{\mu}\right\rangle= & \sum_{n}\left(-\frac{e^{A_{s}-\Phi}}{g_{5}} \partial_{z} v_{a, n}(z)\right) \hat{V}_{a, n}^{\mu}(x), \\
\left\langle J_{A, a}^{\mu}\right\rangle= & \sum_{n}\left(-\frac{e^{A_{s}-\Phi}}{g_{5}} \partial_{z} a_{a, n}(z)\right) \hat{A}_{a, n}^{\mu}(x) \\
& +\sum_{n}\left(-\frac{e^{A_{s}-\Phi}}{g_{5}} \partial_{z} \varphi_{a, n}(z)\right) \partial^{\mu} \hat{\pi}_{a, n}(x) .
\end{aligned}
$$

On the other hand, the meson decay constant are defined by the following relations:

$$
\begin{aligned}
\left\langle 0\left|J_{V, a}^{\mu}(x)\right| V^{b, m}(p, \lambda)\right\rangle & =F_{v^{a, m}} e^{-i p \cdot x} \epsilon^{\mu}(p, \lambda) \delta^{a b}, \\
\left\langle 0\left|J_{A, a}^{\mu}(x)\right| A^{b, m}(p, \lambda)\right\rangle & =F_{a^{a, m}} e^{-i p \cdot x} \epsilon^{\mu}(p, \lambda) \delta^{a b}, \\
\left\langle 0\left|J_{A, a}^{\mu}(x)\right| \pi^{b, m}(p)\right\rangle & =f_{\pi^{a, m}} e^{-i p \cdot x} \delta^{a b} .
\end{aligned}
$$

The quantities $F_{v^{a, m}}, F_{a^{a, m}}$, and $f_{\pi^{a, m}}$ are the decay constants of the vector, axial-vector, and pseudoscalar mesons. Comparing Eqs. (A19) and (A20), we arrive at the holographic dictionary for meson decay constants

$$
\begin{aligned}
F_{v_{a, n}} & =-\frac{e^{A_{s}-\Phi}}{g_{5}} \partial_{z} v_{a, n}(z), \quad F_{a_{a, n}}=-\frac{e^{A_{s}-\Phi}}{g_{5}} \partial_{z} a_{a, n}(z) \\
f_{\pi_{a, n}} & =-\frac{e^{A_{s}-\Phi}}{g_{5}} \partial_{z} \varphi_{a, n}(z) .
\end{aligned}
$$

\section{APPENDIX B: THE GKK MODEL: A REVIEW}

In this section we summarize the model investigated in [11], known as the GKK model (see also [27]). This model was motivated by the original soft wall model [7], which considers a quadratic dilaton from the UV to the IR. In turn, the GKK model proposes reconstructing the dilaton profile by rewriting the tachyon differential equation as

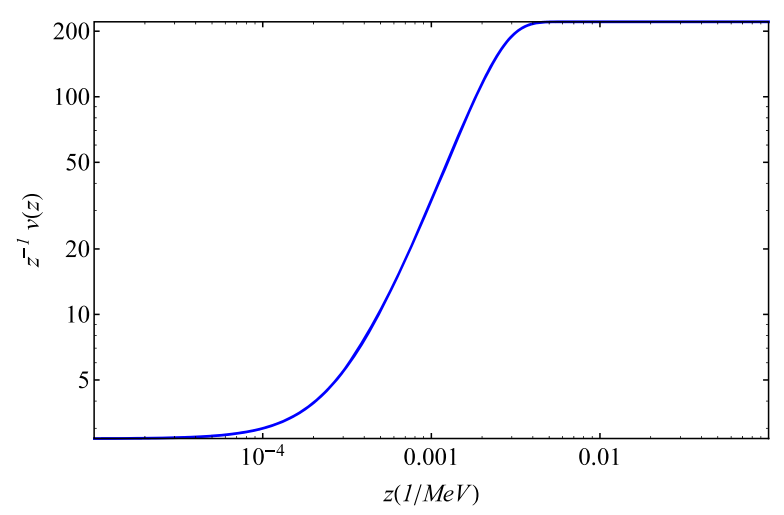

FIG. 25. Profile of the tachyon. To get this figure, we fix the parameters as follows: $\kappa=15, m_{q}=9.75 \mathrm{MeV}, \quad \Sigma=$ $(204.5 \mathrm{MeV})^{3}$ and $\phi_{\infty}=0.1831 \mathrm{GeV}^{2}$. 

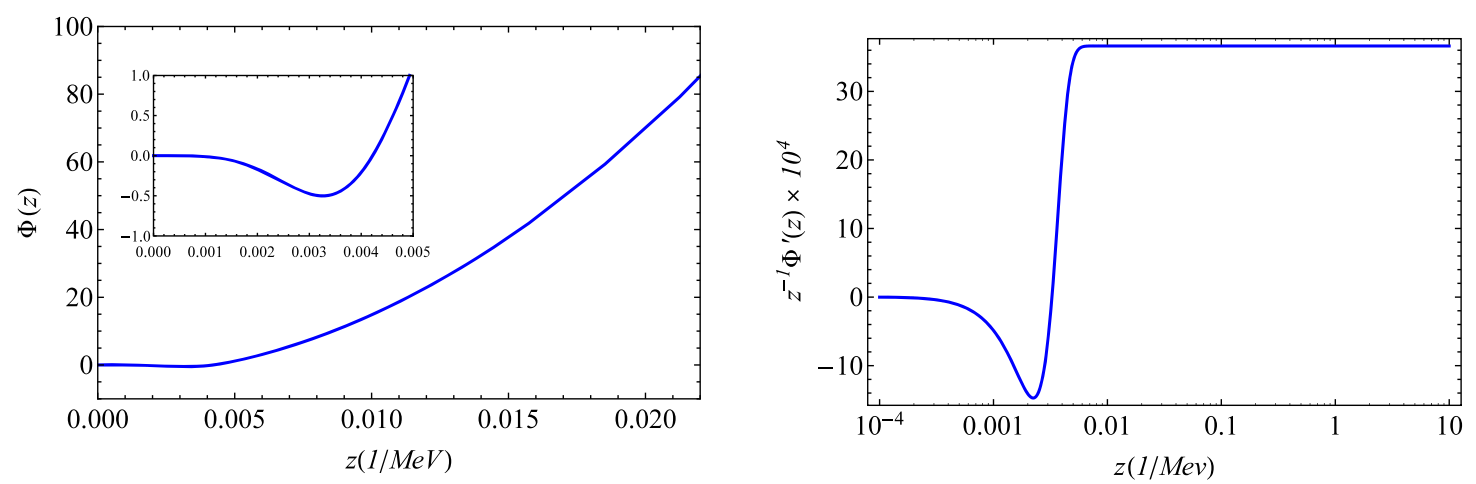

FIG. 26. (Left panel) Profile of the dilaton field. (Right panel) Profile of the derivative of the dilaton. Both results were obtained by setting $\kappa=15, m_{q}=9.75 \mathrm{MeV}, \Sigma=(404.5 \mathrm{MeV})^{3}$, and $\phi_{\infty}=0.1831 \mathrm{GeV}^{2}$.
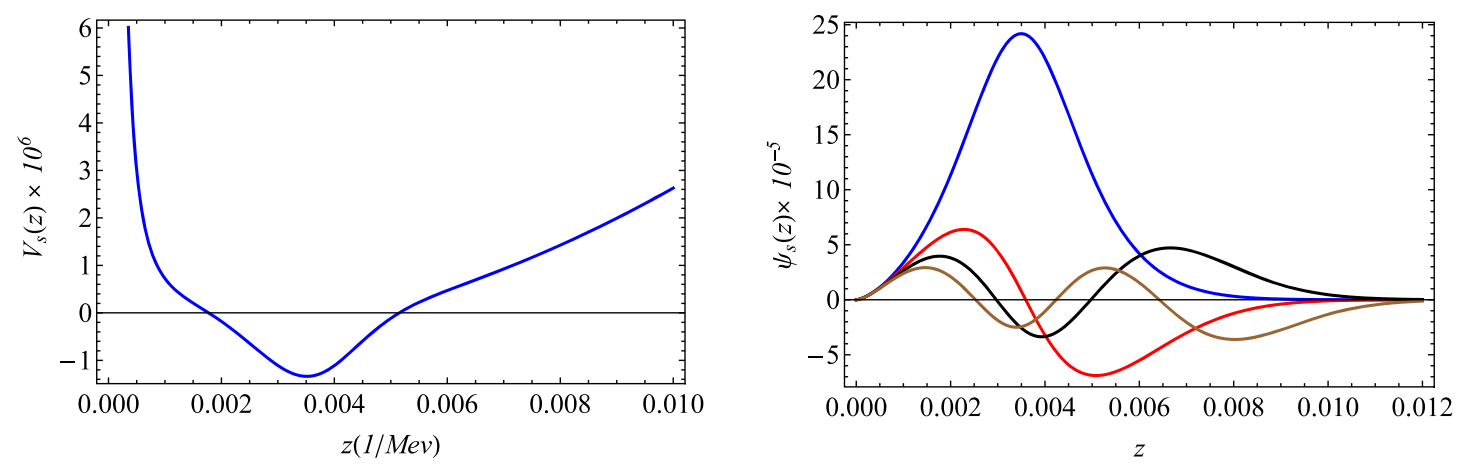

FIG. 27. (Left panel) Potential of the Schrödinger equation associated with the scalar mesons. (Right panel) First wave functions associated with the scalar mesons; see Table XII. Both results were obtained by setting $\kappa=15, m_{q}=9.75 \mathrm{MeV}, \Sigma=(204.5 \mathrm{MeV})^{3}$, and $\phi_{\infty}=0.1831 \mathrm{GeV}^{2}$.

$\partial_{z} \Phi(z)=\frac{\partial_{z}^{2} v}{\partial_{z} v}-e^{2 A_{s}(z)}\left(m_{X}^{2}-\frac{\kappa}{2} v^{2}\right) \frac{v}{\partial_{z} v}+3 \partial_{z} A_{s}(z)$

We observe that this equation depends on the tachyon field. Thus, to solve this equation, we must know the tachyon. On the other hand, the asymptotic expansion of the tachyon field close to the boundary takes the form $v=c_{1} z+c_{3} z^{3}$. In turn, in the IR the tachyon is linearly divergent, $v \sim z$. Additional constraints were imposed by the phenomenology; see [11] for details. Thus, the following interpolation function recovers the asymptotic behavior in the UV and IR:

$$
v(z)=z\left(A+B \tanh \left(C z^{2}\right)\right)
$$

where the parameters of the model are defined as

$$
A=\frac{\sqrt{N_{c}} m_{q}}{2 \pi}, \quad B=2 \sqrt{\frac{\phi_{\infty}}{\kappa}}-\frac{\sqrt{N_{c}} m_{q}}{2 \pi}, \quad C=\frac{2 \pi \Sigma}{\sqrt{N_{c} B}} .
$$

Close to the UV Eq. (B2) takes the form

$$
v(z)=A z+B C z^{3}+\cdots,
$$

where $A \propto m_{q}$ and $B C \propto \Sigma$. In the way the parameters were defined in Eq. (B3), we can see that in the chiral limit, i.e., $m_{q} \rightarrow 0, \Sigma \neq 0$, which means that the spontaneous chiral symmetry breaking is nonzero. In the IR the tachyon reduces to

$$
v(z)=(A+B) z=2 \sqrt{\frac{\phi_{\infty}}{\kappa}} z .
$$

A plot of the tachyon field is shown in Fig. 25. In turn, the dilaton field and its derivative are displayed in the left and right panels of Fig. 26, respectively. As we can see, we notice that the dilaton field becomes negative in a small region close to the boundary. However, regarding the analysis developed in [47], a negative dilaton violates the null energy condition in the gravitational side, where the dilaton must rise monotonically so that $\Phi^{\prime}(z)>0 .{ }^{6}$ Hence, perhaps this

\footnotetext{
${ }^{6}$ It is worth mentioning that the analysis done by Kiritsis and Nitti in [47] states that the dilaton field should be monotonically increasing. However, as the soft wall model is not backreacted, perhaps the null energy condition could be "relaxed" in some manner, at least locally.
} 
TABLE XII. The masses (in MeV) obtained in the modified version of the soft wall model, including the quartic interaction term, compared to the results of [11] and the experimental results [29]. The values of the parameters used are $\kappa=15$, $m_{q}=9.75 \mathrm{MeV}, \Sigma=(404.5 \mathrm{MeV})^{3}$, and $\phi_{\infty}=0.1831 \mathrm{GeV}^{2}$.

\begin{tabular}{lcrc}
\hline \hline$n$ & Model & GKK [11] & $f_{0}$ experimental [29] \\
\hline 1 & $748 \mathrm{i}$ & 799 & $550_{-150}^{+250}$ \\
2 & 799 & 1184 & $980 \pm 10$ \\
3 & 1184 & 1466 & $1350 \pm 150$ \\
4 & 1465 & 1699 & $1505 \pm 6$ \\
5 & 1698 & 1903 & $1724 \pm 7$ \\
6 & 1902 & 2087 & $1992 \pm 16$ \\
7 & 2087 & 2257 & $2103 \pm 8$ \\
8 & 2256 & 2414 & $2314 \pm 25$ \\
\hline \hline
\end{tabular}

pathological behavior will have consequences in the spectrum.

\section{Scalar sector}

We have a special interest in the spectrum of the scalar sector of this model. Now let us compute the spectrum. For doing that, we must rewrite the perturbation equation in the Schrödinger form and solve it using a shooting method, for example. A plot of the potential is shown in the left panel of Fig. 27. The results of the spectrum are displayed in the first column of Table XII, where we see that the first state has an imaginary mass, which indicates an instability, i.e., $m_{s}^{2}<0$. It is worth mentioning that this instability was reported previously in [43] (see also [49]). To guarantee that this state is, in fact, a solution of the Schrödinger equation, we plot the first wave functions in the right panel of Fig. 27. All of these results were obtained using the same parameters as in [11].

\section{APPENDIX C: NUMERICAL ANALYSIS- NONLINEAR SOFT WALL MODEL}

Here we write some details of our numerical results obtained investigating the tachyon field in the model for

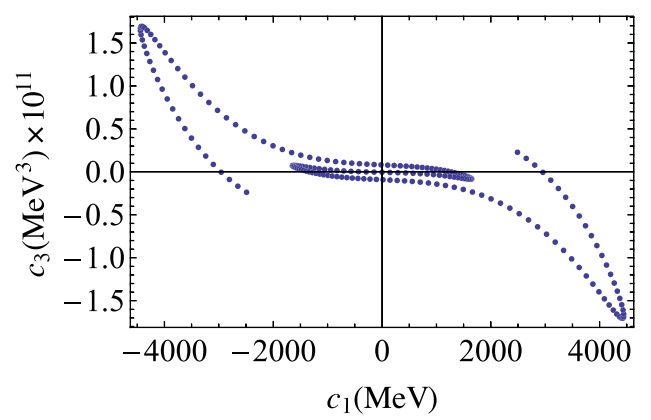

$\lambda<0$. We fix the parameter $\phi_{\infty}=(388 \mathrm{MeV})^{2}$. Then we solve the differential equation (20) numerically while considering a family of two parametric solutions in the UV and a family of one parametric solution in the IR. We use as the boundary condition the asymptotic solution in the IR (26). In the following analysis, we focus in the energy scale in the UV such that the energy belongs to the interval $\left[10^{3}, 10^{6}\right] \mathrm{MeV}$, which is equivalent to the interval of the holographic coordinate $z \in\left[10^{-6}, 10^{-3}\right] \mathrm{MeV}^{-1}$, which lies close to the boundary. Thus, the problem was reduced to solving a one parameter family of solutions in the IR and a two parameter family of solutions in the UV. What is expected is a nontrivial relationship between these parameters, which is obtained by numerically solving Eq. (20). Our numerical results for $c_{3}$ as a function of $c_{1}$ are displayed in Fig. 28 for different values of $\lambda$. From this figure, we observe that there are solutions aside from the trivial solution with $c_{3} \neq 0$ in the chiral limit, i.e., $c_{1} \rightarrow 0$, which corresponds to the limit of spontaneous chiral symmetry breaking.

We point out that, from the set of solutions showed in Fig. 28, the physical solutions are those for which the tachyon field is a monotonic increasing function. The corresponding solutions for $c_{1}$ as a function of $C_{0}$ are displayed in Fig. 29, whereas the solutions for $c_{3}$ as a function of $C_{0}$ are displayed in Fig. 30. However, when computing the spectrum of the vector mesons, for example, we obtain an inconsistency when we calculate the potential of the Schrödinger equation, which is given by

$$
V_{V}=\frac{15}{4 z^{2}}+\phi_{\infty}^{2} z^{2}+2 \phi_{\infty}
$$

At $z_{\min }=10^{-6} \mathrm{MeV}^{-1}$, the first term of the last equation is leading such that $V_{V} \sim\left(10^{10} \mathrm{MeV}\right)^{2}$. On the other hand, at $z_{\max }=10^{-3} \mathrm{MeV}^{-1}$, the first term is still the leading $V_{V} \sim\left(10^{3} \mathrm{MeV}\right)^{2}$, meaning that the potential is a monotonic decreasing function well. We also realized that the convergence of the asymptotic solution (26) is not guaranteed.

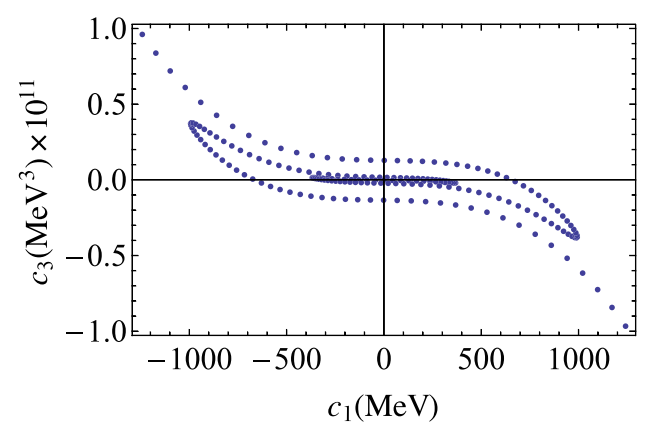

FIG. 28. Numerical results of the nonlinear soft wall model. The corresponding parameters are (left panel) $\lambda=-1$ and (right panel) $\lambda=-20$. 

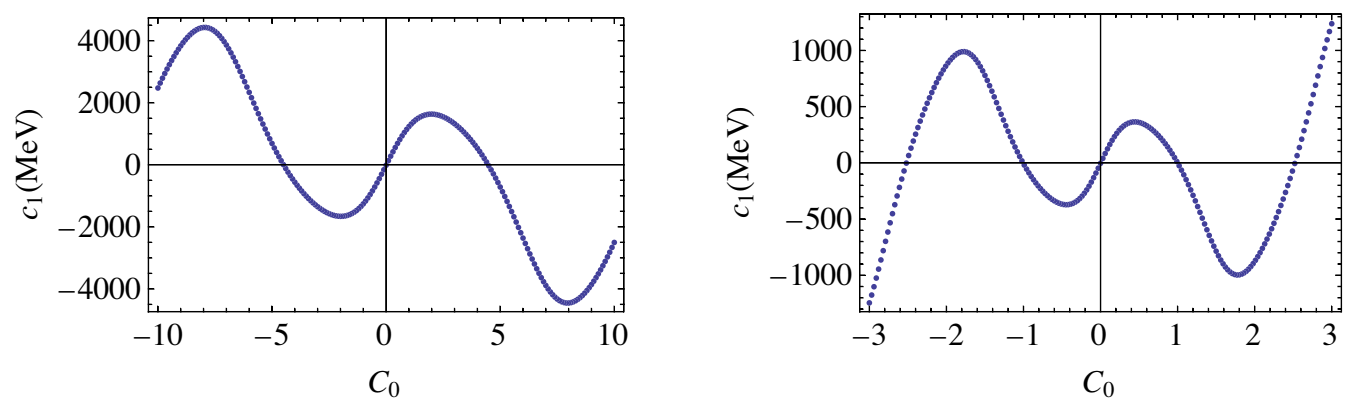

FIG. 29. Numerical results for the nonlinear soft wall model. The corresponding parameters are (left panel) $\lambda=-1$ and (right panel) $\lambda=-20$.
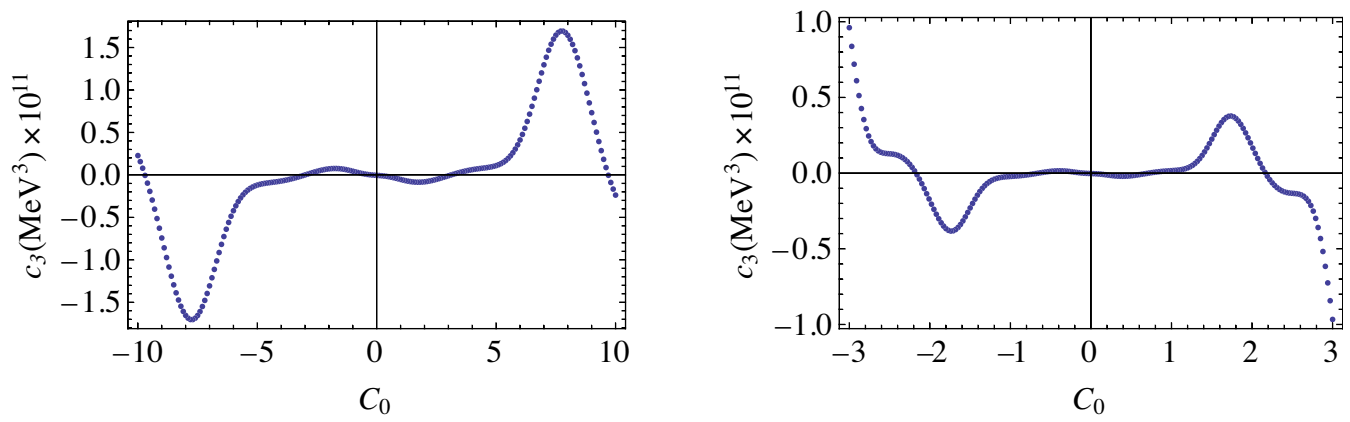

FIG. 30. Numerical results for the nonlinear soft wall model. The corresponding parameters are (left panel) $\lambda=-1$ and (right panel) $\lambda=-20$.

In conclusion, the above analysis shows us that there is spontaneous chiral symmetry breaking in the nonlinear soft wall model for $\lambda<0$. However, it arises in the UV. This conclusion may be justified since the confinement scale introduced by the dilaton field is "fake" because the dilaton is introduced by hand and the backreaction on the metric neglected.

[1] S. R. Coleman and E. Witten, Phys. Rev. Lett. 45, 100 (1980).

[2] Y. Kinar, E. Schreiber, and J. Sonnenschein, Nucl. Phys. B566, 103 (2000).

[3] J. M. Maldacena, Phys. Rev. Lett. 80, 4859 (1998).

[4] J. Erlich, E. Katz, D. T. Son, and M. A. Stephanov, Phys. Rev. Lett. 95, 261602 (2005).

[5] L. Da Rold and A. Pomarol, Nucl. Phys. B721, 79 (2005).

[6] J. Polchinski and M. J. Strassler, Phys. Rev. Lett. 88, 031601 (2002).

[7] A. Karch, E. Katz, D. T. Son, and M. A. Stephanov, Phys. Rev. D 74, 015005 (2006).

[8] C. McNeile, A. Bazavov, C. T. H. Davies, R. J. Dowdall, K. Hornbostel, G. P. Lepage, and H. D. Trottier, Phys. Rev. D 87, 034503 (2013).

[9] K. Chelabi, Z. Fang, M. Huang, D. Li, and Y. L. Wu, J. High Energy Phys. 04 (2016) 036.

[10] I. Iatrakis, E. Kiritsis, and A. Paredes, J. High Energy Phys. 11 (2010) 123.
[11] T. Gherghetta, J. I. Kapusta, and T. M. Kelley, Phys. Rev. D 79, 076003 (2009).

[12] H. Boschi-Filho and N. R. F. Braga, J. High Energy Phys. 05 (2003) 009.

[13] A. Cherman, T. D. Cohen, and E. S. Werbos, Phys. Rev. C 79, 045203 (2009).

[14] C. Csaki and M. Reece, J. High Energy Phys. 05 (2007) 062.

[15] U. Gursoy, E. Kiritsis, and F. Nitti, J. High Energy Phys. 02 (2008) 019.

[16] A. Ballon-Bayona, H. Boschi-Filho, L. A. H. Mamani, A. S. Miranda, and V. T. Zanchin, Phys. Rev. D 97, 046001 (2018).

[17] K. Ghoroku, N. Maru, M. Tachibana, and M. Yahiro, Phys. Lett. B 633, 602 (2006).

[18] P. Colangelo, F. De Fazio, F. Giannuzzi, F. Jugeau, and S. Nicotri, Phys. Rev. D 78, 055009 (2008).

[19] S. J. Brodsky, G. F. de Teramond, H. G. Dosch, and J. Erlich, Phys. Rep. 584, 1 (2015).

[20] O. Bergman, S. Seki, and J. Sonnenschein, J. High Energy Phys. 12 (2007) 037. 
[21] R. Casero, E. Kiritsis, and A. Paredes, Nucl. Phys. B787, 98 (2007).

[22] M. Jarvinen and E. Kiritsis, J. High Energy Phys. 03 (2012) 002.

[23] D. Arean, I. Iatrakis, M. Järvinen, and E. Kiritsis, Phys. Lett. B 720, 219 (2013).

[24] M. Jarvinen, J. High Energy Phys. 07 (2015) 033.

[25] D. Areán, I. Iatrakis, M. Järvinen, and E. Kiritsis, J. High Energy Phys. 11 (2013) 068.

[26] A. Karch, A. O'Bannon, and K. Skenderis, J. High Energy Phys. 04 (2006) 015.

[27] T. M. Kelley, S. P. Bartz, and J. I. Kapusta, Phys. Rev. D 83, 016002 (2011).

[28] C. P. Herzog, Phys. Rev. Lett. 98, 091601 (2007).

[29] M. Tanabashi et al. (Particle Data Group), Phys. Rev. D 98, 030001 (2018).

[30] V. Kaplunovsky and J. Sonnenschein, J. High Energy Phys. 05 (2011) 058.

[31] M. Ihl, M. A. C. Torres, H. Boschi-Filho, and C. A. B. Bayona, J. High Energy Phys. 09 (2011) 026.

[32] L. Da Rold and A. Pomarol, J. High Energy Phys. 01 (2006) 157.

[33] A. Ballon-Bayona, G. Krein, and C. Miller, Phys. Rev. D 91, 065024 (2015).

[34] A. Ballon-Bayona, G. Krein, and C. Miller, Phys. Rev. D 96, 014017 (2017).
[35] H. J. Kwee and R. F. Lebed, J. High Energy Phys. 01 (2008) 027.

[36] H. J. Kwee and R. F. Lebed, Phys. Rev. D 77, 115007 (2008).

[37] A. Holl, A. Krassnigg, and C. D. Roberts, Phys. Rev. C 70, 042203 (2004).

[38] A. Krassnigg, C. D. Roberts, and S. V. Wright, Int. J. Mod. Phys. A 22, 424 (2007).

[39] A. V. Manohar and M. B. Wise, Heavy Quark Physics (Cambridge University Press, Cambridge, England, 2009), https://doi.org/10.1017/CBO9780511529351.

[40] P. Maris and P. C. Tandy, Nucl. Phys. B, Proc. Suppl. 161, 136 (2006).

[41] A. Vega and I. Schmidt, Phys. Rev. D 82, 115023 (2010).

[42] Z. Fang, Y. L. Wu, and L. Zhang, Phys. Lett. B 762, 86 (2016).

[43] Y. Q. Sui, Y. L. Wu, Z. F. Xie, and Y. B. Yang, Phys. Rev. D 81, 014024 (2010).

[44] I. Iatrakis, E. Kiritsis, and A. Paredes, Phys. Rev. D 81, 115004 (2010).

[45] L. X. Cui, Z. Fang, and Y. L. Wu, Eur. Phys. J. C 76, 22 (2016).

[46] A. Karch, E. Katz, D. T. Son, and M. A. Stephanov, J. High Energy Phys. 04 (2011) 066.

[47] E. Kiritsis and F. Nitti, Nucl. Phys. B772, 67 (2007).

[48] Z. Abidin and C. E. Carlson, Phys. Rev. D 80, 115010 (2009).

[49] D. Li and M. Huang, J. High Energy Phys. 11 (2013) 088. 